\title{
Chapter
}

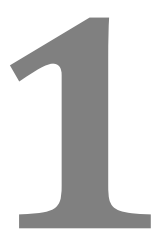

\section{NANOTECHNOLOGY AND ITS IMPLICATIONS IN THERAPEUTICS}

\section{Anuja Patil, Harsiddhi Chaudhary,}

Kisan Ramchandra Jadhav*, and Vilasrao Kadam

University of Mumbai, Bharati Vidyapeeth's College of Pharmacy, Department of Pharmaceutics, CBD Belapur, Sector-8, Navi-Mumbai-400614, India 


\section{Contents}

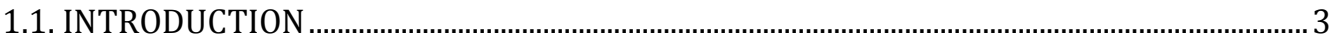

1.2. CURRENT TECHNOLOGIES AND APPLICATIONS OF NANOTECHNOLOGY ................... 10

1.2.1. Polymeric nanomedicine for cancer therapy..............................................................10

1.2.1.1. Nanotechnology in cancer therapy ................................................................11

1.2.1.2. Opportunities and challenges for cancer therapeutics.............................12

1.2.1.3. Passive tumour targeting ................................................................................12

1.2.1.4. Active tumour targeting ..........................................................................13

1.2.1.5. Polymer-based nanomedicine for treating cancer ....................................15

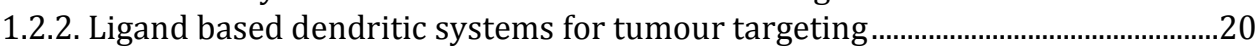

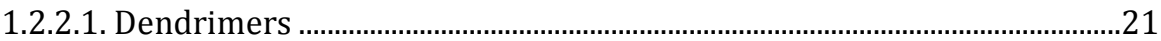

1.2.2.2. Receptor specific dendritic nanoconstructs ..............................................28

1.2.3. Nanomedicine in the diagnosis and therapy of neurodegenerative

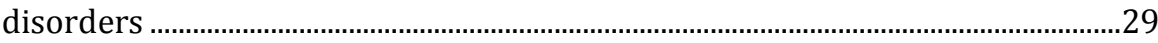

1.2.3.1. Barriers to central nervous system (CNS) drug delivery [102].............29

1.2.3.2. Nanocarriers for CNS drug delivery [103] ..................................................30

1.2.4. Nanoparticle applications in ocular gene therapy …….................................................

1.2.5. Nanoparticles for the treatment of osteoporosis.........................................................38

1.2.6. Applications of nanotechnology in diabetes ..............................................................39

1.2.6.1. Nanomedicine application in glucose and insulin monitoring...............39

1.2.6.2. Nanoparticles in the treatment of diabetes ................................................40

1.2.6.3. Applications of nanotechnology in diabetes.................................................42

1.2.7. Nanosystems in inflammation..........................................................................................4

1.2.7.1. Targeting macrophages to control inflammation .....................................4

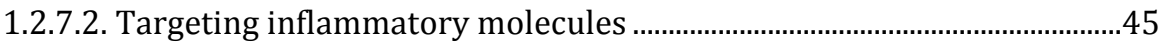

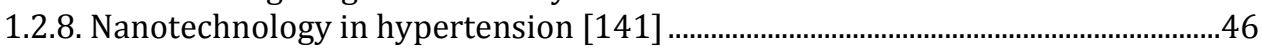

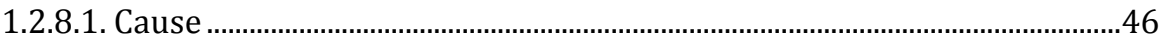

1.2.8.2. Diagnosis and drugs ......................................................................................46

1.2.8.3. Nanoparticle based hypertension treatment.............................................4

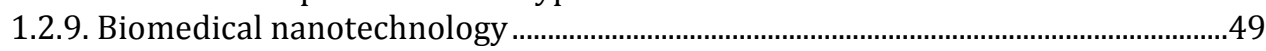

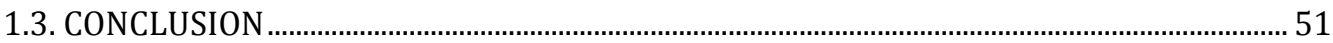

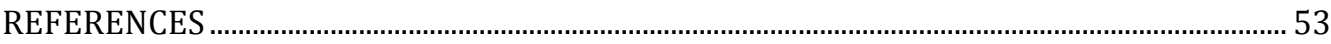




\subsection{INTRODUCTION}

Nanotechnology is the engineering and manufacturing of materials at the atomic and molecular scale resulting into the construction of structures in the nanometre scale size range (often $100 \mathrm{~nm}$ or smaller), without changing unique properties. Indeed, the physical and chemical properties of materials can considerably improve or radically change as their size is reduced to small clusters of atoms. Small size means different arrangements and spacings for surface atoms, and these govern the object's physics and chemistry [1]. The prefix of nanotechnology derives from 'nanos' - the Greek word for dwarf. Nanotechnology is becoming important in fields such as microelectronics, health care, engineering, construction, and agriculture. Recently, the application of nanotechnology in the field of health care is receiving considerable acknowledgement. Today, various treatments are available that are time consuming and are also very expensive, whereas the use of nanotechnology provides quicker and much cheaper treatments.

The field of nanotechnology was first introduced by Professor Richard P. Feynman in 1959 (Nobel laureate in physics, 1965). Nanotechnology has gained the status as one of the critical research endeavours of the early $21^{\text {st }}$ century, as scientists harness the unique properties of atomic and molecular assemblages built at the nanometre scale. The ability to manipulate the physical, chemical, and biological properties of these particles provides researchers with the capability to rationally design and use nanoparticles for drug delivery, as image contrast agents, and for diagnostic purposes [2].

Nanotechnology [3] is a multidisciplinary field covering a large and diverse array of devices and materials in the nanometre scale, derived from engineering, physics, chemistry, and biology. The field of applied nanotechnology in medicine is rapidly growing and the application of nanotechnology to therapeutics has led to advances in drug delivery, biomaterials, biomedical devices, intelligent processes, and many other areas of medicine and applied biomedical sciences.

Nanotechnology is the construction of useful materials, devices and systems used to control matter at an incredibly small scale between 1-1000 nm. Such nanoscale objects can be useful by themselves, or as part of larger devices containing multiple nanoscale objects.

In biopharmaceutical delivery there are a number of features of nanotechnology which make it an appropriate tool to tackle major issues [3-5]:

$>$ Reduction of particle size and increased surface area, enhancing solubility;

$>$ Increasing oral bioavailability;

$>$ Targeting of tissues, cells and cellular receptors; 
$>$ Vaccine and gene delivery;

$>$ Delivery of large macromolecule drugs to intracellular sites of action;

$>$ Co-delivery of two or more drugs or therapeutic moiety for combination therapy;

$>$ Allows passage through biological membranes, especially the blood brain barrier;

$>$ Design of new nanoporous membranes for controlled-release devices;

$>$ Artificial surface engineering of implants to increase biocompatibility;

$>$ Visualisation of sites of drug delivery by combining therapeutic agents with imaging modalities;

$>$ Determining the in vivo efficacy of a therapeutic agent.

Materials used in nanotechnology could be subdivided into several categories:

1. Nanoparticles are solid colloidal particles ranging in size from 1-1000 nm which are comprised of macromolecular materials and used therapeutically; for example, as an adjuvant in vaccines or drug carriers, in which active ingredients are dissolved, entrapped or encapsulated and to which active principle is attached or adsorbed. The chief advantages of nanoparticles are (1) improved bioavailability by enhancing aqueous solubility, (2) increasing residence time in the body (increasing half-life for clearance/increasing specificity for its cognate receptors, and (3) targeting drug to specific location in the body (its site of action). This leads to concomitant reduction in the amount of the drug required and dosage toxicity, thus allowing the safe delivery of toxic therapeutic drugs and the protection of non-target tissues and cells from severe side effects. It is progressively used in different applications, including drug carrier systems and to cross organ barriers such as the blood-brain barrier, cell membrane, etc. The primary manufacturing methods of nanoparticles include the Emulsion-Solvent evaporation method, the Double emulsion and Evaporation method, the Salting out method, the Emulsion-Diffusion method, and the Solvent Displacement/Precipitation method, among others. They are made up of biocompatible lipids and provide sustained effects by either dissolution or diffusion. Nanoparticles can be used in biomedical applications, where they assist laboratory diagnostics, or in medical drug targeting. They are used for in vivo applications such as contrast agents for magnetic resonance imaging (MRI), for tumour therapy or cardiovascular disease [6-8].

2. Nanocages are unique classes of plasmonic nanoparticles with compact sizes varying from 10 to over $150 \mathrm{~nm}$, porous walls, hollow interiors, and easily modified surface chemistry. They are prepared using a remarkably simple galvanic replacement reaction between solutions containing metal precursor salts, mainly chloroauric acid $\left(\mathrm{HAuCl}_{4}\right)$, and $\mathrm{Ag}$ nanostructures prepared 
through polyol reduction. The electrochemical potential difference between the two species allows the reaction, with the reduced metal depositing on the surface of the Ag nanostructures. The resultant $\mathrm{Au}$ is deposited epitaxially on the surface of the Ag nanocubes, adopting their underlying cubic form. Concomitant with this deposition, the interior Ag is oxidised and removed, together with alloying and dealloying, to produce hollow and, ultimately, porous structures commonly known as Au nanocages. This approach is versatile; based upon the initial shape of an Ag template, a vast range of morphologies (e.g. nanorings, prism shaped nanoboxes, nanotubes, and multi walled nanoshells or nanotubes) are available. This novel class of hollow nanostructures is finding its use as both a contrast agent for optical imaging in the early stages of tumour detection, and as a therapeutic agent for photothermal cancer treatment. Gold nanocages can target tumour cells in vitro by complexing with antibodies through the gold thiolate-linkage. When coated with smart thermosensitive-polymer, gold nanocages can also serve as drug delivery vehicles, emptying their contents in response to near-infrared irradiation [6,9-11].

3. Nanocomposites are materials that are formed by introducing nanoparticulates (often referred to as filler) into a macroscopic sample material (often referred to as the matrix). This is part of the growing field of nanotechnology. After adding nanoparticulates to the matrix material, the resulting nanocomposite may manifest drastically enhanced properties. For example, adding carbon nanotubes tends to significantly add to the electrical and thermal conductivity. Depending upon the matrix materials, nanocomposites can be classified as ceramic matrix nanocomposites (CMNC), metal matrix nanocomposites (MMNC), polymer matrix nanocomposites (PMNC). CMNC matrix materials include $\mathrm{Al}_{2} \mathrm{O}_{3}, \mathrm{SiC}, \mathrm{SiN}$, etc., while metal matrices employed in $\mathrm{MMNC}$ are mainly $\mathrm{Al}, \mathrm{Mg}, \mathrm{Pb}, \mathrm{Sn}, \mathrm{W}$ and $\mathrm{Fe}$, and a whole range of polymers, e.g. vinyl polymers, condensation polymers, polyolefins, speciality polymers (including a variety of biodegradable molecules) are used in PMNC. Processing methods for CMNC include the Powder Process, the Polymer Precursor process, and the sol-gel process, while processing methods for MMNC include Spray Pyrolysis, Liquid Infiltration, Rapid Solidification Process (RSP) with ultrasonication, High Energy Ball Milling, Chemical Processes (Sol-gel, Colloidal), etc. Important methods of synthesis for PMNC are intercalation of nanoparticles with the polymer or pre-polymer from solution, in situ intercalative polymerisation, melt intercalation, direct mixture of polymer and particulates, template synthesis, in situ polymerisation, sol-gel process, etc. These nanocomposites are useful in numerous areas ranging from packaging to biomedical applications $[6,12,13]$.

4. Nanofibres are defined as fibres with a diameter of less than $100 \mathrm{~nm}$. They can be prepared by interfacial polymerisation, electrospinning and phase separation techniques. Carbon nanofibres are graphitised fibres produced by catalytic synthesis. The development of nanofibres has improved the scope for 
fabricating scaffolds that can potentially resemble the architecture of natural human tissue at the nanometre scale. The high surface area to volume ratio of the nanofibres coupled with their microporous structure promotes cell adhesion, proliferation, migration, and differentiation, all of which are highly desired properties for tissue engineering applications. Because of their potential, the nanofibre-based systems are also being sought for a variety of other biological and non-biological applications. Currently, nanofibres are finding use as scaffolds for musculoskeletal tissue engineering (including bone, cartilage, ligament, and skeletal muscle), skin tissue engineering, neural tissue engineering, vascular tissue engineering, and controlled delivery of drugs, proteins, and DNA $[6,14]$.

5. Nanorings are small rings formed of crystal. A zinc-oxide nanoring was the first nanoring discovered by researchers. They are synthesised by a spontaneous self-coiling process of nanobelts. Many layers of nanobelts are rolled together as coils, layer-by-layer. The seamless nanorings, each made of a uniformly deformed single crystal of zinc oxide, could be used for nanoscale devices and serve as a model system for studying electrical and mechanical coupling at the nanoscale. These rings, which range in diameter from 1-4 microns and which are $10-30 \mathrm{~nm}$ thick, form in a horizontal tube furnace when a mixture of zinc oxide, indium oxide and lithium carbonate - at a ratio of $20: 1: 1$ - is heated to $1,400^{\circ} \mathrm{C}$ under a flow of argon gas [6].

6. Nanorods are one type of morphological nanoscale objects. Each of their dimensions range from $1-100 \mathrm{~nm}$. They may be prepared from metals or semiconducting materials. Nanorods are produced by direct chemical synthesis. One potential application of nanorods is in display technologies, because the reflectivity of the rods can be changed by altering their orientation with an applied electric field. Another application is for microelectromechanical systems (MEMS). Nanorods, along with other noble metal nanoparticles, also serve as theragnostic agents. Nanorods absorb in the near infrared (IR), and generate heat when excited with IR light. This property makes nanorod a useful nanotool in cancer therapeutics. Nanorods can be conjugated with tumour-targeting motifs and ingested. When a patient is exposed to IR light (which passes through body tissue), nanorods selectively taken up by tumour cells are locally heated, destroying only the cancerous tissue, while protecting the healthy cells [6].

7. Nanoshell consists of a spherical core of a particular compound enclosed by a shell of a few nm in thickness. Currently, gold nanoshells (AuNSs) are being investigated as nanocarriers for drug delivery systems and have both diagnostic as well as therapeutic applications, together with photothermal ablation, hyperthermia, and drug delivery, and diagnostic imaging, particularly in oncology. Localised surface plasmon resonance, biocompatibility, low immunogenicity, and facile functionalisation make AuNSs valuable nanocarriers. AuNSs used for drug delivery can be spatially and temporally triggered to release controlled quantities of drugs inside the target cells when 
illuminated with a near-infrared (NIR) laser. Recently, many research groups have confirmed that these AuNS complexes are able to carry anti-tumour drugs (e.g. doxorubicin, paclitaxel, small interfering RNA, and single-stranded DNA) into cancer cells, which augment the efficacy of the treatment. AuNSs can also be conjugated with active targeting ligands such as antibodies, aptamers, and peptides to increase the particles' specificity towards the desired targets [6,15].

8. Quantum dots (QDs) are tiny light-emitting particles on the nanometre scale, and are emerging as a new class of fluorescent labels for biology and medicine. QDs are novel type of semiconductor nanocrystals made up of an inorganic elemental core (e.g. cadmium, mercury) surrounded by a metal shell. Two universal approaches for the preparation of QDs have been reported over the last decade: (1) the formation of nanosized semiconductor particles through colloidal chemistry and (2) epitaxial growth and/or nanoscale patterning i.e. employing lithography-based technology. As compared to organic dyes and fluorescent proteins, they cover unique optical and electronic properties, with size-tunable light emission, superior signal brightness, resistance to photobleaching, and broad absorption spectra for the simultaneous excitation of multiple fluorescence colours. QDs also provide a versatile nanoscale scaffold for designing multifunctional nanoparticles with both imaging and therapeutic functions. When functionalised with targeting ligands such as antibodies, peptides or small molecules, QDs can be used to target tumour biomarkers as well as tumour vasculatures with high affinity and specificity. QDs can be used as imaging probes for both in vitro and in vivo cellular and molecular imaging $[6,16,17]$.

9. Fullerenes are a class of allotropes of carbon which are graphene sheets rolled into tubes or spheres. The structures of fullerenes can be designated as symmetric cages of all $\mathrm{sp}^{2}$ carbons, which belong to either 5- or 6-member rings on the cage surface. Fullerene cages of different sizes $\left(C_{60}, C_{70}\right.$, and so on) consist of these rings in different numbers and ratios. It has been deduced that size, hydrophobicity, three-dimensionality and electronic configurations make them an attractive entity in medicinal chemistry. Their distinct carbon cage structure coupled with immense scope for derivatisation make them a potential therapeutic agent. The fullerene family, and especially $\mathrm{C}_{60}$, has appealing photo, electrochemical and physical properties, which can be used in various medical fields. The geometry of fullerene matches with the shape of hydrophobic cavity of human immunodeficiency virus (HIV) proteases, thus making them able to fit inside the cavity and hence inhibiting the access of substrates to the catalytic site of the enzyme. It can be used as a radical scavenger and antioxidant. At the same time, when exposed to light, fullerene can produce singlet oxygen in high quantum yields. This action, and also the direct electron transfer from excited states of fullerene and DNA bases, altogether can be used to cleave DNA. Additionally, fullerenes have been used as a carrier for gene and drug delivery systems $[6,18,19]$. 
10. Carbon Nanotubes (CNTs) are allotropes of carbon with a nanostructure that can have a length-to-diameter ratio greater than 1,000,000. They display extraordinary strength and unique electrical properties, and are efficient conductors of heat. Inorganic nanotubes have also been synthesised. Nanotubes are classified as single-walled nanotubes (SWNTs) and multi-walled nanotubes (MWNTs). Single-walled boron-nitride (BN) nanostructures are hypothetically stronger and lighter than steel. Once BN nanostructures are surrounded by polymers, they can serve to ruggedise the surface of metal parts, as well as forming the basis for oxidation-proof coating. The three most commonly used methods for the synthesis of carbon nanotubes includes the arc-discharge method, the laser ablation method and the chemical vapour deposition techniques. Their remarkable structural, mechanical, and electronic properties are because of their small size and mass, their strong mechanical potency, and their high electrical and thermal conductivity. CNTs have been successfully used in pharmacy and medicine due to their high surface area, which is able to adsorb or conjugate with a wide variety of therapeutic and diagnostic agents (drugs, genes, vaccines, antibodies, biosensors, etc.). For the first time, they have been demonstrated as an excellent vehicle for drug delivery directly into cells without metabolism by the body. Other applications of CNTs include tissue regeneration, biosensor diagnosis, enantiomer separation of chiral drugs, extraction and analysis of drugs and pollutants. Recently, CNTs have been revealed to be promising antioxidants $[6,20]$.

Application of nanotechnology in areas of drug delivery and therapy has the potential to revolutionize the treatment of many diseases. In the past two decades, several nanotherapeutics were approved by Food and Drug Administration (FDA) for the treatment of cancer, pain, and infectious diseases (Table 1). Advanced therapy can only be achieved through the rational design of nanotherapeutics that leads to the development of nanoplatforms of particular size, shape, and surface properties that are crucial for biological interactions and consequent therapeutic effects. In the market, nanotherapeutic products include nanocrystals, liposomes, nanoemulsions, nanocomplexes, and nanoparticles, as listed in Table 1 . The majority of nanotherapeutics on the market is intended for parenteral administration. These are followed by nanotherapeutics designed for oral administration. The choice of the delivery route, and consequently the barriers to be crossed, are of particular importance for drug-delivery systems [21]. 
Table 1. Marketed nanotechnology based formulations

\begin{tabular}{|c|c|c|c|c|}
\hline $\begin{array}{l}\text { Nanotechnology } \\
\text { based approach }\end{array}$ & Drug & $\begin{array}{c}\text { Major } \\
\text { indication }\end{array}$ & $\begin{array}{l}\text { Dosage } \\
\text { form }\end{array}$ & Brand name \\
\hline \multirow[t]{4}{*}{ Nanocrystals } & Sirolimus & $\begin{array}{l}\text { Graft rejection, } \\
\text { Kidney } \\
\text { transplantation }\end{array}$ & Tablet & Rapamune ${ }^{\circledR}$ \\
\hline & Fenofibrate & $\begin{array}{c}\text { Hyper- } \\
\text { cholesterolemia }\end{array}$ & Tablet & $\begin{array}{c}\text { Tricor }{ }^{\circledR} / \\
\text { Lipanthyl }{ }^{\circledR} / \\
\text { Lipidil }{ }^{\circledR}\end{array}$ \\
\hline & Aprepitant & $\begin{array}{c}\text { Postoperative } \\
\text { nausea and } \\
\text { vomiting, Cancer }\end{array}$ & Capsule & Emend $^{\circledR}$ \\
\hline & Olanzapine & Schizophrenia & $\begin{array}{l}\text { Powder and } \\
\text { solvent for } \\
\text { prolonged } \\
\text { release } \\
\text { suspension } \\
\text { for injection }\end{array}$ & Zypadhera ${ }^{\circledR}$ \\
\hline \multirow[t]{2}{*}{ Nanoemulsion } & Cyclosporine & $\begin{array}{l}\text { Prophylaxis of } \\
\text { organ rejection } \\
\text { following organ } \\
\text { transplant }\end{array}$ & Soft capsule & Neoral $^{\circledR}$ \\
\hline & Ritonavir & HIV Infection & Soft capsule & Norvir ${ }^{\circledR}$ \\
\hline \multirow[t]{5}{*}{ Liposome } & Amphotericin B & Fungal infection & $\begin{array}{l}\text { Suspension } \\
\text { (iv) }\end{array}$ & AmBisome ${ }^{\circledR}$ \\
\hline & Daunorubicin & $\begin{array}{l}\text { Cancer advanced } \\
\text { HIV-associated } \\
\text { Kaposi's } \\
\text { sarcoma }\end{array}$ & $\begin{array}{l}\text { Suspension } \\
\text { (iv) }\end{array}$ & DuanoXome $^{\circledR}$ \\
\hline & Doxorubicin & $\begin{array}{l}\text { Breast } \\
\text { Neoplasms } \\
\text { Kaposi's } \\
\text { sarcoma } \\
\end{array}$ & $\begin{array}{l}\text { Suspension } \\
\text { (iv) }\end{array}$ & Myocet ${ }^{\circledR}$ \\
\hline & Cytarabine & $\begin{array}{l}\text { Meningeal } \\
\text { neoplasms }\end{array}$ & $\begin{array}{l}\text { Suspension } \\
\text { (intrathecal) }\end{array}$ & DepoCyt $^{\circledR}$ \\
\hline & Propofol & Anaesthetic & $\begin{array}{l}\text { Emulsion } \\
\quad \text { (iv) }\end{array}$ & $\begin{array}{c}\text { Diprivan }^{\circledR} \\
\text { Propofol- } \\
\text { Lipuro }^{\circledR} \\
\end{array}$ \\
\hline Nanoparticles & Paclitaxel & $\begin{array}{c}\text { Breast } \\
\text { neoplasms }\end{array}$ & $\begin{array}{l}\text { Powder for } \\
\text { suspension } \\
\text { for infusion }\end{array}$ & Abraxane ${ }^{\circledR}$ \\
\hline Nanocomplex & $\begin{array}{l}\text { Sodium ferric } \\
\text { gluconate }\end{array}$ & $\begin{array}{l}\text { Iron deficiency } \\
\text { anaemia }\end{array}$ & $\begin{array}{l}\text { Solution } \\
\text { (iv) }\end{array}$ & Ferrlecit $^{\circledR}$ \\
\hline
\end{tabular}




\subsection{CURRENT TECHNOLOGIES AND APPLICATIONS OF NANOTECHNOLOGY}

\subsubsection{Polymeric nanomedicine for cancer therapy}

As the world population ages, the incidence of cancer is increasing continuously; regardless of the tremendous efforts to treat cancer, there has been very little actual improvement in cancer therapeutics over the past few years. Nanomedicine, a branch of nanotechnology, refers to highly specific, molecular-scale medical intervention for treating disease or repairing damaged tissues [22,23]. The nanomedicine-based diagnostics such as gold nanoshells, iron oxide nanocrystals, and quantum dots have gained tremendous impetus in the diagnosis of cancer, although their practical application has been limited by problems such as toxicity, instability, and lack of selectivity for the disease site. Recently, researchers have provided technical solutions to overcome these limitations by physically or chemically attaching biocompatible polymers on the surfaces of diagnostic nanomedicines. Most of the polymers used for these systems are biocompatible and/or biodegradable, and are thus approved by the FDA. The drug is usually either dispersed within the polymeric nanoparticle or conjugated with the polymeric backbone. In the former case, the encapsulated drugs are slowly released from the polymer matrix by diffusion. In the latter case, surface erosion or bulk degradation of the polymer matrix can play a primary role in drug release, and various techniques may be used to adjust the release rate. Several natural and synthetic water soluble polymers and their derivatives have been investigated for their potential use in polymer therapeutics, including poly(ethylene glycol) (PEG), $N$-(2-hydroxypropyl) methacrylamide copolymers, poly(vinyl pyrrolidone), chitosan, hyaluronic acid, poly(ethyleneimine) (PEI), dextran and poly(aspartic acid) [24,25]. Polymer-based nanomedicine (Figure 1), mainly includes the use of polymer-DNA complexes (polyplexes), polymer-drug conjugates [26] and polymer micelles [27] bearing hydrophobic drugs, and has gained increasing consideration due to its ability to improve the efficacy of cancer therapeutics [28]. Due to their small size and excellent biocompatibility, nanosized polymer therapeutic agents can remain in the bloodstream for longer duration, hence allowing them to reach the target site. Additionally, chemical modification of polymer therapeutic agents by the use of ligands which are capable of specifically binding receptors that are over-expressed in cancer cells can markedly enhance therapeutic efficiency. 


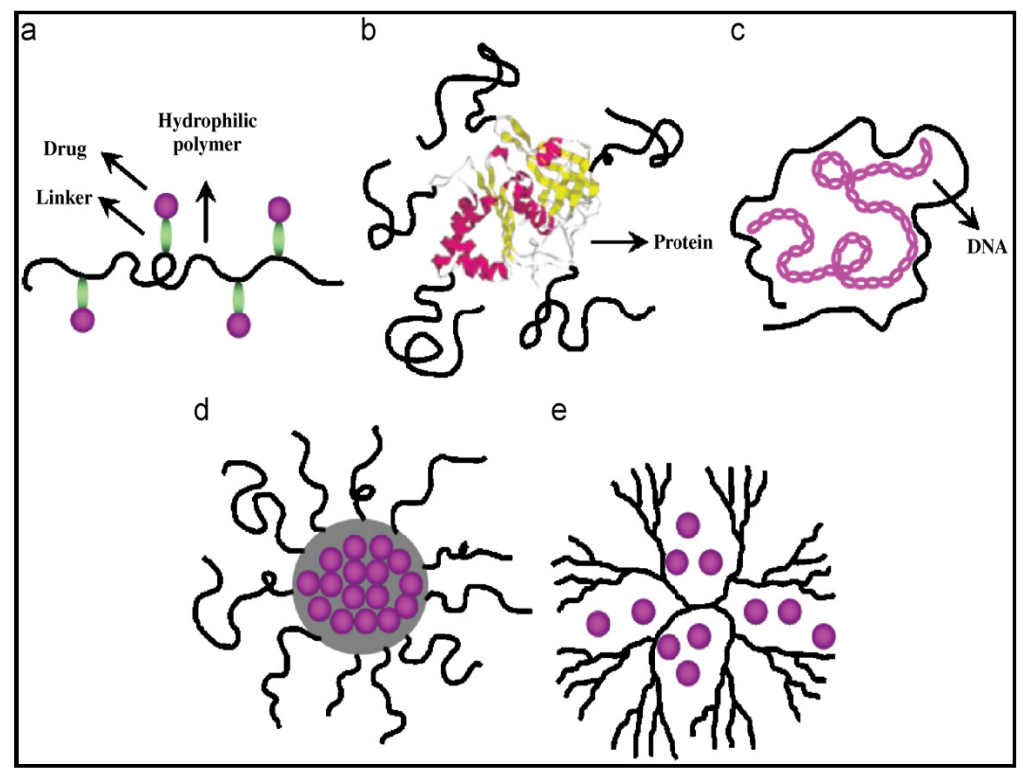

Figure 1. Schematic illustration of representative polymeric nanomedicines:

(a) polymer-drug conjugates; (b) polymer-protein conjugates; (c) polymer-DNA complexes; (d) polymeric micelles; (e) dendrimers

\subsubsection{Nanotechnology in cancer therapy}

Current nanotechnologies in cancer chemotherapy include the following issues [29]:

$\checkmark$ Conventional anticancer drugs, when administered intravenously, can lead to the distribution of drugs throughout the entire body via the bloodstream, and also affect both malignant as well as normal cells, thus producing adverse effects.

$\checkmark$ The interstitium of a tumour is characterised by high hydrostatic pressure which is opposite to that of most normal tissues, leading to an outward convective interstitial flow that can wash the drug away from the tumour.

$\checkmark$ Although the drug is successfully delivered to the tumour interstitium, its efficacy may be hampered by the cancer cells that have acquired multidrug resistance (MDR) [30]. MDR is mainly characterised by over-expression of the plasma membrane P-glycoprotein (P-gp), which is able to drive the drugs away from the cell. Several strategies have been used to circumvent P-gp-mediated MDR, which includes the co-administration of P-gp inhibitors and anchoring the anticancer drugs within the nanoparticles. The latter strategy should allow the drug to avoid recognition by P-gp at the plasma membrane, enabling its delivery to the cell cytoplasm or nucleus [31]. 


\subsubsection{Opportunities and challenges for cancer therapeutics}

Angiogenesis in cancer [32-35]

In the case of cancer, solid tumours [36] smaller than 1-2 $\mathrm{mm}^{3}$ are not vascularised because oxygen and nutrients can reach the centre of the tumour by simple diffusion. Moreover, solid tumours larger than the critical volume of $2 \mathrm{~mm}^{3}$ enter a state of cellular hypoxia that marks the onset of tumoral angiogenesis, i.e. the developing of new blood vessels from existing vessels.

A fine balance between factors capable of stimulating and inhibiting blood vessel formation regulates angiogenesis. This balance tips in favour of angiogenesis when hypoxia induces the cancer cell to release pro-angiogenic molecules such as growth factors.

Various strategies for interfering in the angiogenic process have been investigated, including the inhibition of endogenous angiogenic factors (e.g. growth factors), degradative enzymes [e.g. matrix metalloproteases (MMPs)] which digest the extracellular matrix and allow the blood vessels to form towards the tumour tissue and endothelial cell processes (e.g. differentiation, activation, migration, and proliferation), which are necessary for angiogenesis. Moreover, the tumour vasculature caused by angiogenesis in cancer is morphologically abnormal, and various cell-surface proteins have been associated with promoting angiogenesis. Thus, it should be possible to selectively destroy tumour neovasculature devoid of significantly affecting normal vessels.

In terms of nanomedicine, recently prepared nanocells are polymer-based nuclear nanoparticle embedded within an extranuclear PEGylated lipid envelope. This nanocell bears temporal release of two therapeutic agents wherein an anti-angiogenic agent is released from the outer envelope, blocking vascularisation, after which a chemotherapeutic agent is released from the inner nanoparticle to kill the cancer cells. This study confirmed that the combination of traditional chemotherapy with anti-angiogenic agents in a polymeric nanoparticular system improved the therapeutic index while reducing toxicit.

\subsubsection{Passive tumour targeting}

Most anticancer drugs used in conventional chemotherapy have no tumour selectivity and are randomly distributed within the body, resulting in a reasonably low therapeutic index. Furthermore, recent studies have shown that polymer-conjugated drugs and nanoparticulates show prolonged circulation in the blood and accumulate passively in tumours, even in the absence of targeting ligands, suggesting the existence of a passive retention mechanism. Tumour blood vessels are generally denoted by abnormalities such as a relatively high proportion of proliferating endothelial cells, increased tortuosity, pericyte deficiency and aberrant basement membrane formation. 
This defective vascular structure, which is probably the result of the rapid vascularisation necessary to provide oxygen and nutrients for fast-growing cancers, reduces lymphatic drainage and renders the vessels permeable to macromolecules. Due to the reduction in the lymphatic drainage, the permeant macromolecules are not removed efficiently, and are thus maintained in the tumour. This passive targeting phenomenon (Figure 2) is known as the enhanced permeation and retention (EPR) effect $[37,38]$.

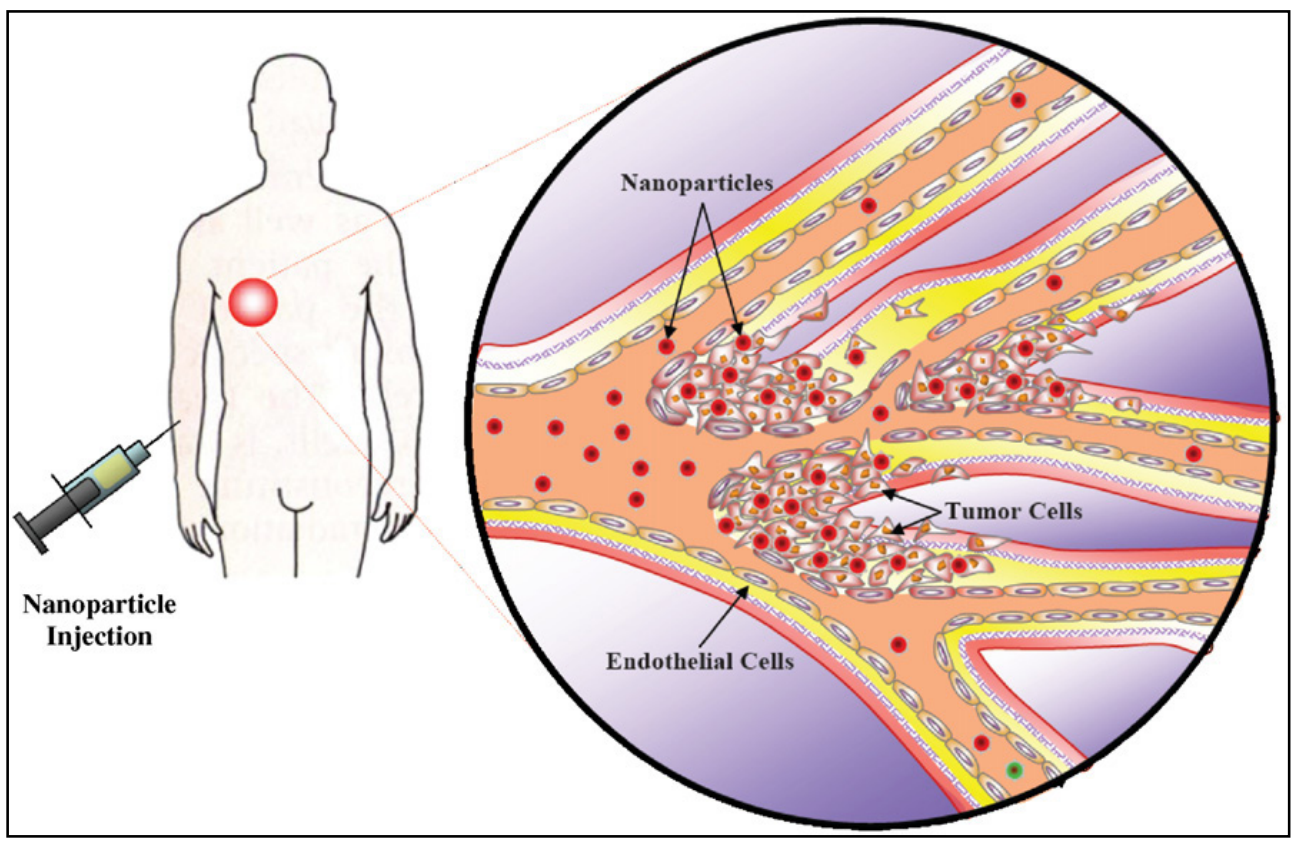

Figure 2. Passive drug targeting through the enhanced permeability and retention (EPR) effect. The polymeric nanoparticles preferentially accumulate in solid tumors, owing at least in part to leaky tumor vessels and an ineffective lymphatic drainage system.

\subsubsection{Active tumour targeting}

Active drug targeting is typically achieved by chemical attachment to a targeting component that strongly interacts with antigens (or receptors) present on the target tissue, resulting into the preferential accumulation of the drug in the targeted organ, tissue, or cells. The use of a targeting moiety not only reduces adverse side effects by allowing the drug to be delivered to the specific site of action, but also facilitates cellular uptake of the drug by receptor-mediated endocytosis, which is an active process requiring a significantly lower concentration gradient across the plasma membrane than 
simple endocytosis. Active targeting frequently makes use of monoclonal antibodies. However, nowadays folate targeting is preferred.

Folates [39-41] are low molecular weight vitamins that are mandatory for eukaryotic cells and their conjugates have the ability to deliver a variety of drugs or imaging agents to pathological cells without causing harm to normal tissues. (Figure 3).

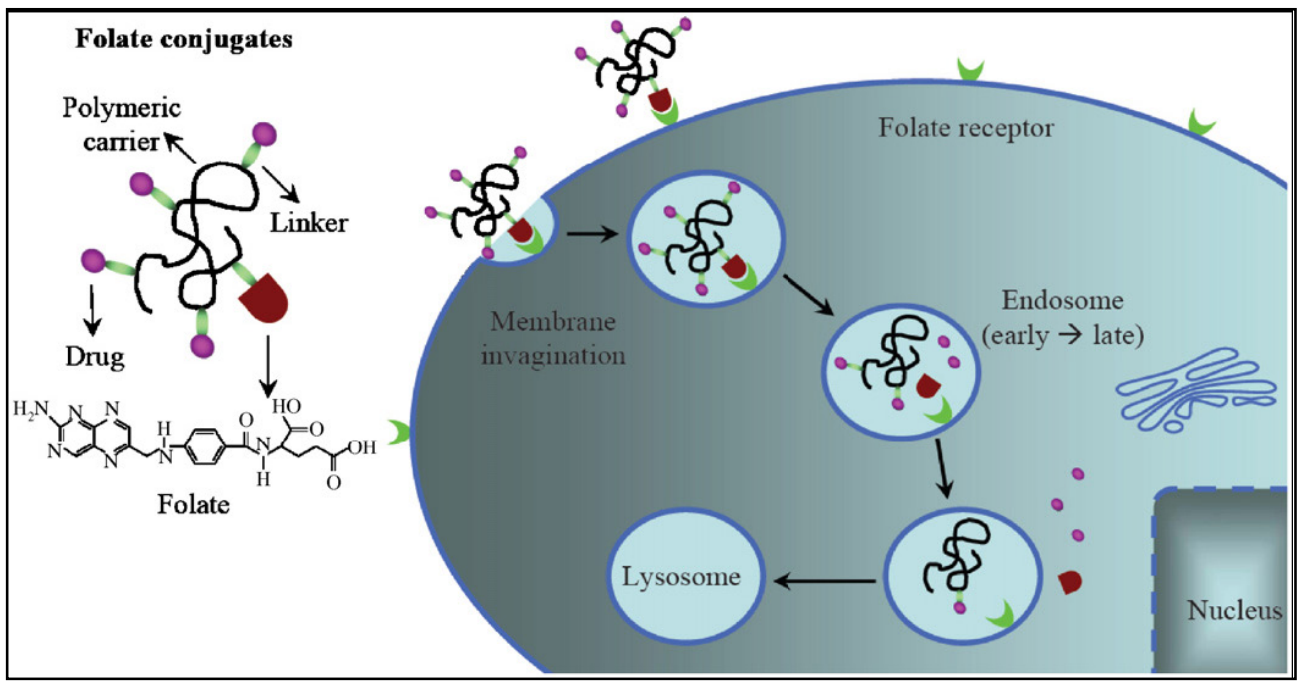

Figure 3. Receptor-mediated endocytosis of folate-conjugated drugs. The folate receptors recognize the conjugates, which are subsequently subjected to membrane invagination. As the endosomal compartment acidifies, the conjugate and the drugs are released from the receptor into the cytosol.

Advantages of folate targeting:

1. Folate is known to be non-immunogenic.

2. Folate-conjugated drugs or nanoparticles are very quickly internalised via receptor-mediated endocytosis.

3. In addition, the use of folate as a targeting moiety is believed to evade cancer cell multidrug-efflux pumps [41]. 


\subsubsection{Polymer-based nanomedicine for treating cancer}

Effective gene therapy requires two vital components [42], namely:

1. An efficacious therapeutic gene that can be expressed at a target cell; and

2. A safe and efficient delivery system that can transport the therapeutic gene to the specific tissue or organ.

Two different types of carriers have been examined for use in delivering genes to the target site: "viral and non-viral vectors".

Viral vectors, which cover retroviruses, adenoviruses, and adeno-associated viruses [43], are capable of introducing their genetic materials into the host cells, which results in high gene transfection efficiency.

Limitations of viral vectors:

1. The large genetic materials required for gene therapy are difficult to encapsulate;

2. Risks based on their immunogenicity and oncogenic potential [44, 45].

Thus, non-viral vectors, especially polymers, have recently turned out to be a promising alternative that exhibits considerably lower safety risks and can be tailored to specific therapeutic needs through relatively simple changes in the preparation, purification and chemical modification steps [46,47]. Genetic material of various sizes can be delivered by non-viral vectors, and can be prepared easily and inexpensively. However, when compared to viral vectors, they show relatively low transfection efficiency and a short duration of gene expression.

The most commonly used non-viral vectors include polymeric gene carriers $[42,48]$ and polymer micelles.

\section{Polymeric gene carriers}

\section{Poly(ethyleneimine) (PEI)}

Most of the polymer-based non-viral vectors are cationic in nature and can interact electrostatically with negatively charged DNA to produced nanosized ionic complexes (polyplexes) [49-51]. In general, polyplexes reveal optimal transfection efficiency when they have a positive net charge generated by the presence of more a cationic polymer than DNA [51]. This allows the polyplexes to interact efficiently with the negatively charged cell surface proteoglycans that mediate subsequent endocytosis [52]. Erythrocyte aggregation and/or interaction with plasma components such as albumin and fibrinogen can occur upon the intravenous administration of positively charged polyplexes. Polyplexes, when chemically conjugated to PEG or various Targeting moieties, can minimise such problems. 
There are several factors affecting transfection efficiency and cytotoxicity of PEI-based polyplexes:

1. The molecular weight of the PEI [53],

2. The ionic strength of the solution,

3. Its degree of branching,

4. The zeta potential of the polyplexes and

5. Their particle size, confirming that low molecular weight PEI (LMW-PEI, $11.9 \mathrm{kDa}$ ) produces a higher transfection efficiency and lower cytotoxicity than high molecular weight PEI (HMW-PEI, $1616 \mathrm{kDa}$ ) [53].

Researchers have chemically conjugated PEG to the PEI backbone. It is usually accepted that the surface modification of biomolecules with PEG leads to increased blood circulation time [54], because PEG appreciably decreases the uptake of the polyplexes by macrophages in the liver and spleen through a phenomenon called the "stealth effect". Other advantages of PEG are:

1. PEG is a biocompatible polymer;

2. Cytotoxicity is decreased when it is conjugated with polyplexes;

3. Greater transfection efficiency.

In recent years, PEGylated polyplexes bearing ligands capable of targeting specific cells are prepared, as this leads to an increase in both the blood circulation time and the transfection efficiencies of the polyplexes. These efforts may be broadly divided into three different approaches [55]:

(1) PEG is chemically grafted to PEI, such that the PEG functional groups are located at the end of the chain, thus enabling chemical conjugation of the ligands (Figure 4a).

(2) polyplexes prepared by mixing PEI and DNA are modified with heterobifunctional PEG, followed by the chemical attachment of the ligands (Figure 4b).

(3) PEI has been ligand-modified and used to form polyplexes, which are then surface-decorated with PEG (Figure 4c).

Out of the three approaches, the first two have shown better transfection efficiencies, most probably because the ligand is conjugated with the distal end of the PEG, which has more access to the target cells. 


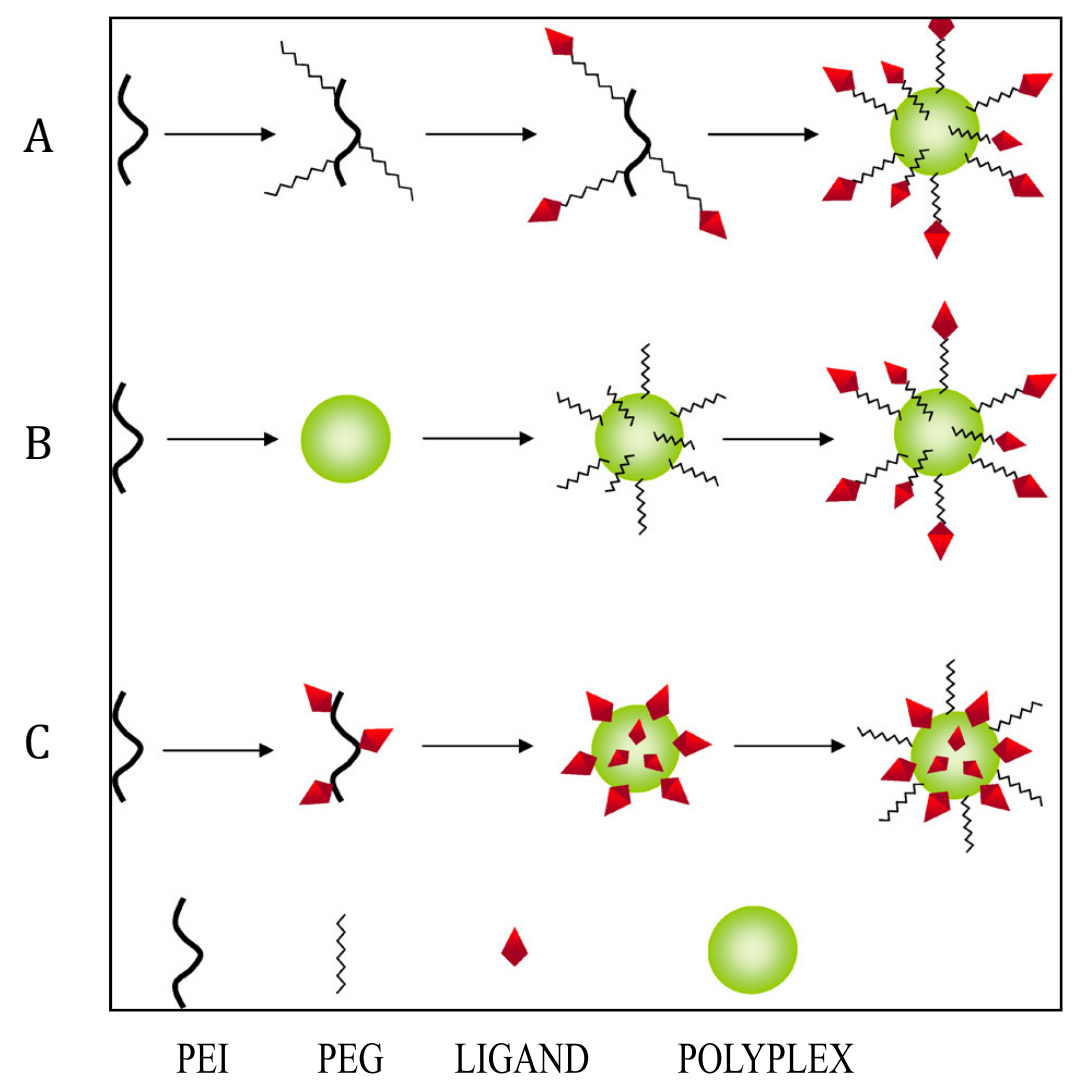

Figure 4. Schematic representation of three strategies used for the formation of PEGylated ligand-containing PEI/DNA complexes

Poly(L-lysine) (PLL)

PLL, which forms polyelectrolyte complexes with DNA, is another polymer that can be used as a non-viral gene carrier. PLL is a linear polypeptide comprising of repeated lysine residues, which have primary e-amino groups and are protonated in the physiological environment. The cationic nature of PLL enables it to interact electrostatically with negatively charged DNA, forming nanoparticulate polyplexes that exhibit different properties depending on the molecular weight of the utilised PLL. The LMW PLLs $(<3 \mathrm{kDa})$ do not form many stable complexes with DNA. The HMW versions of PLL can form nanosized complexes with DNA, but show relatively high toxicities, and the complexes tend to aggregate in aqueous solution. To address such problems, researchers have modified PLL with PEG and various targeting moieties [56]. PEGylation is expected to improve the stability in addition to pharmacokinetic properties of polyplexes. 


\section{Synthetic biodegradable polycations}

Ideally, a gene carrier should not only carry the gene to specific cells with high efficacy, it should also degrade and be excreted from the body after a given time period. Since the non-degradable cationic polymers (e.g. PEI) are not easily removed from the body, they may accumulate within cells or tissues, leading to serious side effects. In an effort to overcome these issues, researchers have assessed the use of biodegradable polycations, such as poly $(\alpha$-(4-aminobutyl)-L-glycolic acid) (PAGA) [57], poly( $\beta$-amino ester) [58], poly(4-hydroxy-L-proline ester), poly(phosphoester) [59], polyphosphazene and degradable PEI, as gene carriers.

PAGA, which showed 2-fold higher transfection efficiency than PLL without any measurable cytotoxicity, showed rapid initial degradation within $100 \mathrm{~min}$ and complete degradation within 6 months in physiological buffer $(\mathrm{pH} \mathrm{7.3)}$ at $37.1^{\circ} \mathrm{C}$. In contrast to PAGA, after 3 months under the same conditions, PLL showed negligible degradation. Poly(amino ester), prepared by the addition of primary amines to diacrylate esters, showed lower cytotoxicity and transfection efficiency and was found to be similar to unmodified PEI.

\section{Polymer micelles}

Polymeric micelles [60], which were introduced by Ringsdorf in 1984, are formed by amphiphilic block copolymers in aqueous solution [61]. Polymeric micelles are capable of enhancing the solubility of hydrophobic drugs stems from their unique structural composition, which is characterised by a hydrophobic inner core sterically stabilised by a hydrophilic shell. A polymeric micelle can serve as a nanosized container into which drugs can be incorporated by chemical, physical, or electrostatic interactions [62].

The use of polymeric micelles as drug carriers offers several advantages over conventional dosage forms:

1. The protection of drugs from harsh biological environments (e.g. low $\mathrm{pH}$ and hydrolytic enzymes);

2. The agents can be imbibed into the hydrophobic inner core, appreciably improving the aqueous solubility of hydrophobic drugs;

3. The small size of polymeric micelles $(10-100 \mathrm{~nm}$ in diameter) should facilitate drug targeting and reduce the side effects of chemotherapy;

4. They have prolonged retention time in circulation. The presence of hydrophilic polymers on the surfaces of nanoparticulate systems is known to hamper protein adsorption and opsonisation of the particles by the reticulo-endocytic system (RES). 
PEG-poly(amino acid) [63-65]

PEG has been widely used as the hydrophilic segment of polymeric micelles. Due to their biocompatibility and hydrophilic nature, PEG-based polymeric micelles have shown no significant cytotoxicity and are not often recognised by the RES system, allowing prolonged circulation in the bloodstream. The PEG chains of polymeric micelles possess high chain mobility in an aqueous environment and have a large excluded volume, potentially decreasing the interactions of the polymeric micelles with constituents of biological fluids. In addition, the PEG molecules in the outer layer of the polymeric micelles can inhibit hydrophobic interactions between the inner cores of different micelles, thus blocking inter-particle aggregation. When amphiphilic block copolymers are prepared with heterobifunctional PEG with different functional groups, the polymeric micelles can be modified with targeting moieties for drug delivery to specific cells and/or tissues.

Poly(ethylene oxide)-block-poly(L-amino acid) [PEO-b-p(L-AA)s] is the modified version of the PEG-poly(amino acids). In addition to loading of therapeutic substances by both chemical and physical means, the use of PEO-b-p(L-AA) also facilitates chemical modification of the core-forming blocks. The $\mathrm{p}(\mathrm{L}-\mathrm{AA}) \mathrm{s}$ are biodegradable, biocompatible and relatively nontoxic. They undergo hydrolysis and/or enzymatic degradation in biological fluids to produce biocompatible L-amino acid materials.

\section{PEG-polyester}

Biocompatible polyesters have been widely used for drug delivery, because they are slowly degraded in the body and thus an additional removal procedure after implantation is not necessary. They are also useful for the preparation of amphiphilic block copolymers that are capable of forming micelles in aqueous solutions. The representative polyesters that can be used as the hydrophobic segments of the copolymers include poly(glycolic acid), poly(D-lactic acid), poly( $\varepsilon$-caprolactone) (PCL), and poly(D, L-lactic acid), as well copolymers of lactide/glycolide.

A model for the cellular internalisation of the drug incorporated in PCL-b-PEO micelles [66-67] has been proposed by the researchers. They proposed that:

(i) by means of endocytosis, the micelle bearing the drugs enters the cytoplasmic compartment;

(ii) from micelle-incorporated drugs, the drug molecules eventually diffuse out of the micelle and distribute through the cytoplasm; and

(iii)Some of the micelles inside the cell may disassemble into single chains and act locally to penetrate the membranes of cellular organelles (Figure 5). 


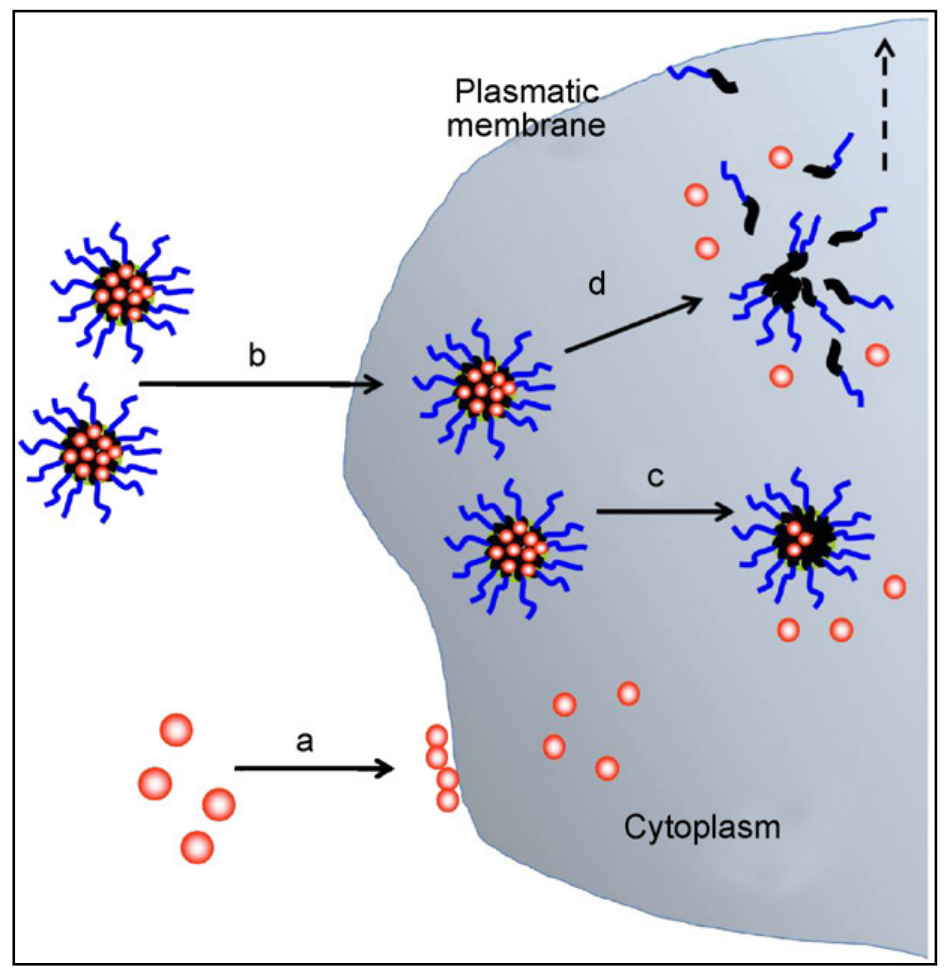

Figure 5. Cellular internalization of free drug and drug incorporated in PCL-b-PEO micelles: (a) free drug diffuses through the cell membrane; (b) micelle bearing the drug enters the cytoplasmic compartment by endocytosis; (c) eventually diffuses out of the micelle and distributes through the cytoplasm; and (d) some of the micelles inside the cell may disassemble into single chains and act locally to permeabilize the membranes of the cellular organelles (dotted arrows)

\subsubsection{Ligand based dendritic systems for tumour targeting}

Medications that can selectively target tumours whilst at the same time avoiding access of the drug to non-target areas employ the use of homing devices termed ligands that can bind to specific epitopes expressed on the surface of the necrotic mass of cells. Dendrimers [68] are nanosized, non-immunogenic, and hyper-branched vehicles that can be efficiently tailored for the spatial distribution of bioactives, thereby reducing untoward cytotoxicity on normal cells. These nanoparticulate drug delivery vehicles provide a unique platform that has exactly placed functional groups so that multiple copies of ligands can be attached to them and facilitate targeting to the tumour surface or neo-vascularising vessels proliferating around these cells. 


\subsubsection{Dendrimers}

Dendrimers are uni-molecular polymeric systems synthesised in a re-iterative manner. At the same time, their synthesis can be optimised to control their size, shape, molecular mass, composition and reactivity. Dendrimers have hyper-branched structure with precisely placed functional groups that bear an important role in controlling the properties of therapeutic moieties that are encapsulated or complexed with it.

\section{Types of dendrimers}

\section{1) Poly (amidoamine) (PAMAM) dendrimer}

These were the first dendritic structures to have been exhaustively investigated. Tomalia's PAMAM dendrimer received widespread attention. PAMAM dendrimers are synthesised by the divergent method starting from ammonia or ethylenediamine initiator core reagents. Products up to generation 10 (a molecular weight of over 930,000 $\mathrm{g} \mathrm{mol}^{-1}$ ) have been obtained. The polydispersity index of 5.0-10.0 G PAMAM dendrimers is less than 1.08, indicating that the particle size distribution is very uniform for each generation. PAMAM dendrimers have the ability for condensation of DNA followed by transfection due to the presence of positive charge on the surface [69].

\section{2) Poly(propylenimine) (PPI) dendrimer}

PPI dendrimers are hyper-branched macromolecules that are amine terminated. The divergent method is basically used to synthesise the PPI dendrimers [70]. Nitrogen of primary amine and nitrogen of tertiary amine are two types of nitrogen atoms in a PPI dendrimer. Tertiary nitrogen atoms are more acidic, with a pKa of around 6-9, whereas primary nitrogen atoms are more basic, with a pKa of around 10. PPI dendrimers are synthesised by a divergent approach, in a sequence of repetition of double Michael addition of acrylonitrile to primary amines followed by heterogeneously catalysed the hydrogenation of nitriles. This repeated reaction results in a doubling of the number of primary amines. 1,4-Diaminobutane is utilised as a dendrimer core during the synthesis of PPI dendrimers. A variety of molecules with primary or secondary amine groups can also be used as the core in dendrimer synthesis [71].

\section{3) Liquid crystalline (LC) dendrimers}

Mesogenic LC monomers e.g. mesogen functionalised carbosilane dendrimers are found in LC dendrimers. Rod-like (calamitic) or disk-like (discotic) molecules form thermotropic LC phases or mesophases [72]. 
Frey and coworkers attached several mesogenic units to carbosilane dendrimers, such as cyanobiphenyl [73] and cholesteryl [74]. Mesogenic 3,4-bis-(decyloxy)benzoyl groups functionalised PPI dendrimers of different generations (1.0-5.0 G) were investigated for mesogenic activity as per the reported study. Apart from the fifth generation dendrimer, all other lower generation dendrimers displayed a hexagonal columnar mesophase in which the dendrimers had a cylindrical conformation [75,76]. The fifth generation dendrimer lacks mesomorphism. This lack of mesomorphism for the fifth generation dendrimer was due to its inability to reorganise into a cylindrical shape. Boiko et al. reported the debut synthesis of photosensitive LC dendrimer with terminal cinnamoyl groups in 2001 [77]. These LC dendrimers are being investigated by scientists for biomedical applications. Recently, Pedziwiatr-Werbicka and coworkers suggested that amino terminated carbosilane dendrimers have potential to deliver short-chain siRNA and anti-HIV oligodeoxynucleotide to HIV-infected blood cells. Although these dendrimers had limited application in the delivery of long-chain double stranded nucleic acids, the dendriplexes of carbosilane dendrimers and anti-HIV nucleic acid were stable and less cytotoxic to blood cells compared to the plain dendrimers, suggesting their utility in the delivery of bioactives.

\section{4) Core Shell (tecto)-dendrimers}

Core-shell or tecto-dendrimers represent a polymeric architecture with highly ordered structure. This highly ordered structure was obtained as a result of the controlled covalent attachment of dendrimer building blocks [78]. Tecto-dendrimers are composed of a core dendrimer that may or may not contain the therapeutic agent, surrounded by dendrimers. The synthesis of tecto-dendrimers has been reported with fluorescein as the core reagent for detection and folate as the targeting moiety [79]. The solubility problems encountered in previous studies with aromatic fluorescein isothiocyanate (FITC) moieties on dendrimeric surfaces were solved by such conjugates. This conjugate was found to be overwhelmingly superior to those dendrimeric conjugates containing both FITC and folic acid attached to the surface [80]. In contrast to simple dendrimers, the synthesis procedure for tecto-dendrimers is comparatively simple and thus later inflating the application of dendrimers. Schilrreff et al. investigated the cytotoxicity of tecto-dendrimers in order to point out their application in biomedical fields. In this study, tecto-dendrimers with amine-terminated 5.0 G PAMAM dendrimers as a core, surrounded by a shell composed of $2.5 \mathrm{G}$ PAMAM dendrimers with surface carboxyl groups, were investigated for cytotoxicity towards SK-Mel-28 human melanoma cells. The inhibition of growth of melanoma cells occurred at a concentration which is safe to healthy keratinocytes epithelial cells [78] with these tecto-dendrimers. Hence, tecto-dendrimers could be explored for application in the field of nanomedicine, including drug delivery. 


\section{5) Chiral dendrimers}

Dendrimers based upon the construction of constitutionally different but chemically similar branches to the chiral core are referred to as chiral dendrimers. Chiral, non-racemic dendrimers with well-defined stereochemistry are a particularly interesting subclass with potential applications. These have applications in asymmetric catalysis and chiral molecular recognition. Ghorai et al. described the first molecules of anthracene-capped chiral dendrimers which were derived from a 1,3,5-trisubstituted aromatic core and carbohydrate units in the interior and periphery. These were claimed to be suitable for anchoring other useful functionalities aimed at applications as drug delivery system and light harvesting materials [81]. Evidence supporting the above claim is keenly awaited, particularly in the field of drug delivery application of chiral dendrimers.

\section{6) Peptide dendrimers}

Peptide dendrimers are radically branched macromolecules that contain a peptidyl branching core and/or peripheral peptide chains. Peptide dendrimers can be divided into three categories. The first category with peptides only as surface functionalities is referred to as grafted peptide dendrimers. The second category composed entirely of amino acids is known as peptide dendrimers, while the third one utilises amino acids in the branching core and surface functional groups but having non-peptide branching units. The synthesis of peptide dendrimers is the best and frequently performed by divergent and convergent methods. The availability of solid-phase combinatorial methods facilitates large libraries of peptide dendrimers to be produced and screened for desired properties. Peptide dendrimers have been used in industry as surfactants and in biomedical field as multiple antigen peptides (MAP), protein mimics and vehicles for drug and gene delivery $[82,83]$. In addition, Darbre and Reymond have utilised peptide dendrimers as esterase catalysts [84].

\section{7) Glycodendrimers}

Glycodendrimers encompass sugar moieties such as glucose, mannose, galactose and/or disaccharide into their structure. The vast majority of glycodendrimers have saccharide residues on their outer surface. Glycodendrimers containing a sugar unit as the central core, from which all branches emanate, have also been described. Glycodendrimers are generally divided into three categories: i) carbohydrate-centred, ii) carbohydrate-based, and iii) carbohydrate-coated dendrimers [85]. One anticipated application of these dendrimers is site-specific drug delivery to the lectin-rich organs. These dendrimers were anticipated to display better association with lectin-supported systems compared to mono-carbohydrate-anchored systems [86]. 


\section{8) Hybrid dendrimers}

Hybrid dendrimers are a combination of dendritic and linear polymers in hybrid block or graft copolymer forms. The formation of dendritic hybrids is due to the spherical shape and a large number of surface functional groups of dendrimers. The small dendrimer segment coupled to multiple reactive chain ends provides an opportunity to use them as surface active agents, also as compatibilisers or adhesives, or hybrid dendritic linear polymers. The dendritic hybrids obtained from various polymers with dendrimers generated the compact, rigid, uniformly shaped globular dendritic hybrids that have been explored for various aspects in the field of drug delivery $[87,88]$.

\section{9) PAMAM-organosilicon (PAMAMOS) dendrimers}

Inverted unimolecular micelles that consist of hydrophilic, nucleophilic PAMAM interiors and hydrophobic organosilicon (OS) exteriors are known as radially layered PAMAMOS dendrimers (PAMAMOS). PAMAMOS dendrimers offer unique potential for novel application in electronics, chemical catalysis, nano-lithography, photonics, etc. It is possible due to its unique properties such as constancy of structure and ability to form complex and encapsulate various guest species with nanoscopic topological precision [89].

\section{Synthesis of dendrimers}

Dendrimers are symmetrical, highly branched polymers possessing a compact spherical structure (diameter ranging from $1.1 \mathrm{~nm}$ for 1.0 G PAMAM to $9 \mathrm{~nm}$ for $8.0 \mathrm{G}$ PAMAM dendrimer). They are normally synthesised from a central polyfunctional core which is achieved by the repetitive addition of monomers. The core is characterised by a number of functional groups. The addition of monomers to each functional group results in next dendrimer generation, as well as the expression of end groups for further reaction [90]. The size of the dendrimer increases as the generation number increases. A stage will soon be reached when the dendrimer attains its maximum size and becomes tightly packed, giving the appearance of a ball. Divergent and convergent methods are most frequently used for dendrimer synthesis. Additionally, other approaches like hypercores and branched monomers growth, double exponential growth, lego chemistry and click chemistry are also used.

\section{1) Divergent approach}

The divergent approach is comprised of two steps. First, is the activation of functional surface groups, and second is the addition of branching monomer units. The approach includes reacting the core with two or more moles of reagent containing at least two protecting/branching sites, followed by the removal of protecting groups. This will lead to the formation of first generation dendrimers. The process is then repeated several times until the dendrimer of 
the desired size is formed. This method gives PAMAM starburst dendrimers. Compared to other methods, the divergent approach has some overriding advantages such as the ability to modify the surface of dendrimer molecules by changing the end groups at the outermost layer. The fact that the overall chemical and physical properties of dendrimer can be configured to specific needs is the other major advantage.[91,92].

\section{2) Convergent approach}

The convergent approach is an alternative method of dendrimer synthesis that was first proposed by Hawker and Frechet in 1990. Only one kind of functional group on the outermost generation is the main constraint of divergent growth method. Convergent growth would overcome such a weakness. Convergent method involves two stages, firstly a reiterative coupling of protected/deprotected branch to produce a focal point functionalised dendron; and secondly, divergent core anchoring steps to produce various multidendron dendrimers. Some outstanding dividends of this method are the precise control over molecular weight and the production of dendrimers with functionalities in precise positions and number are some of the outstanding dividends of this method [93]. A significant limitation of this method is the difficulty in synthesising dendrimers in large quantities, because of repeated, reactions occurring during the convergent approach that necessitates the protection of active sites.

\section{3) Hypercores and branched monomers}

The pre-assembly of oligomeric species to hasten up the rate of dendrimer synthesis is the requirement of this method. Oligomeric species are linked together to yield dendrimers in fewer steps and/or higher yields in this method. Essentially, a hypercore with multiple attaching groups is grown from a core molecule and the surface units are linked to a branched monomer with focal point activation, leading to the synthesis of blocks that are then attached to the hypercore to generate higher generation dendrimers.

\section{4) Double Exponential}

This approach allows the preparation of monomers for both divergent and convergent growth from a single starting material. The starting material is similar to a rapid growth technique for linear polymer. The two resultant products are then reacted to give an orthogonally protected trimer that can be used to repeat the growth again. The advantage of the double exponential growth approach is rapid synthesis and also the applicability to either the divergent or convergent method [94]. 


\section{5) Lego chemistry}

Various approaches have been explored by scientists in order to simplify the synthetic procedure for dendrimers, in terms of cost as well as duration of synthesis. Lego chemistry is one of the outcomes of these explorations. Lego chemistry is based on the application of highly functionalised cores and branched monomers. It has been utilised in the synthesis of phosphorus dendrimers. The basic synthetic scheme has undergone several modifications and has resulted in a refined scheme wherein a single step can amplify the number of terminal surface groups from 48-250. This method also encompasses the advantage of utilising the minimum volume of solvent, allowing a simplified purification procedure with eco-friendly by-products like water and nitrogen, apart from higher growth in the number of terminal surface groups in fewer reactions [95].

\section{6) Click chemistry}

Another approach for the fast and reliable synthesis of dendrimers is based on click chemistry. In click chemistry, small units are joined together. High chemical yield with innocuous by-products is the main characteristic of the click chemistry reaction. The use of simple reaction conditions, easily available reagents, and benign solvent are the additional profits of click chemistry. Following the click chemistry strategy, dendrimers with various surface groups can be obtained in high purity and excellent yield. $2.0 \mathrm{G}$ and $3.0 \mathrm{G}$ triazole dendrimers were synthesised using $\mathrm{Cu}(\mathrm{I})$-catalysed click chemistry reactions. The obtained dendrimers were isolated as a pure, solid sample with only sodium chloride as the major by-product using chromatographic procedure [95].

\section{Dendrimer Generations}

Dendrimer generation is hyperbranching when going from the centre of the dendrimer towards the periphery, which results in homostructural layers between the focal points (branching points). The number of focal points when going from the core towards the dendrimer surface is the generation number, i.e. a dendrimer with five focal points when going from the centre to the periphery is denoted a $5^{\text {th }}$ generation dendrimer. This term, here abbreviated to simply a G5-dendrimer, e.g. a $5^{\text {th }}$ generation PPI, is abbreviated to a "G5-PPI"-dendrimer. The core part of the dendrimer is sometimes denoted generation "zero", or presented as "G0", in the terminology. As hydrogen substituents are not considered focal points, the core structure thus presents no focal points. Intermediates during the dendrimer synthesis are sometimes denoted half-generations $[96,97]$. 
Properties of dendrimers [98]:

(1) Monodispersive nature;

(2) Globular shape;

(3) Efficient carrier system for drugs due to their highly controlled architecture;

(4) Highly stable carriers and can be stored for longer periods;

Dendrimers consist of three characteristic scaffolds:

(1) Multifunctional initiator core;

(2) Inner generations, which are composed of repeating branched units; and

(3) The outermost generation with attached exterior surface groups (Figure 6)

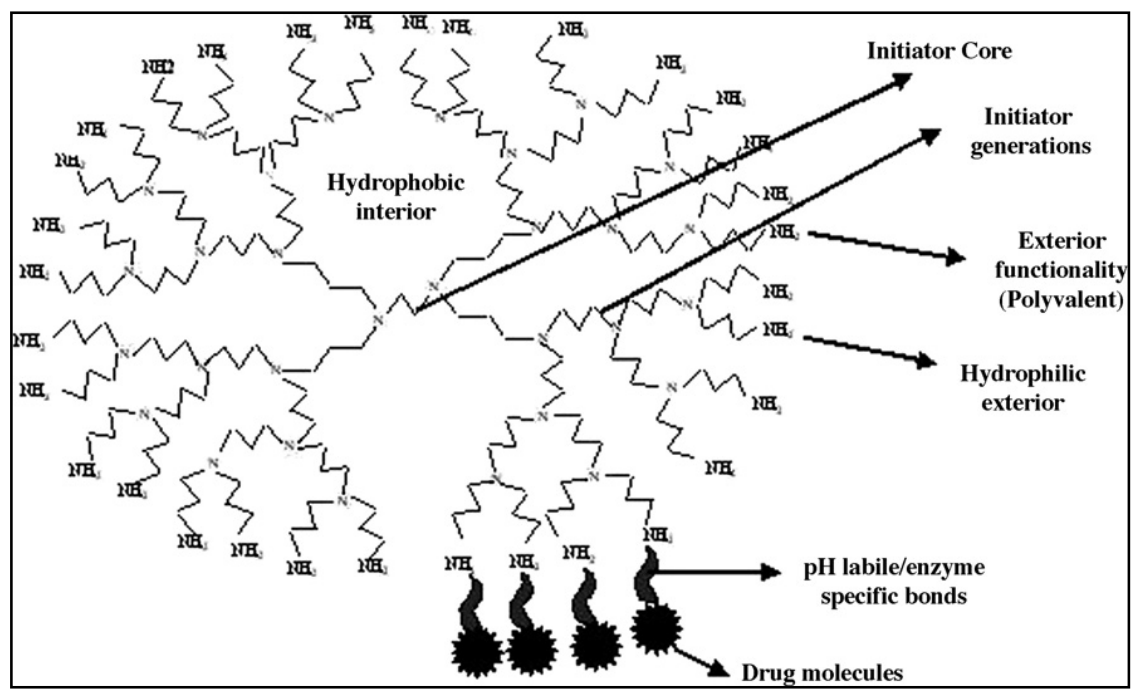

Figure 6. Dendrimer: Unique architecture facilitates (a) encapsulation, (b) conjugation of drugs, (c) prevent their opsonisation by mononuclear phagocytic system (MPS), and (d) provides numerous sites of attachment

The term "exo-receptors" means the terminal functionalities of the dendrimers, which are involved in complexation of therapeutic moieties, whereas the term "endo-receptors" means groups present in the interior responsible for drug entrapment. Dendrimers may have the same functional group at the terminal junctions, which can be an important feature for substrate binding to these functionalities. Multivalency provided by the 
dendrimer can play a superior role in the increased affinity of substrates to its complementary receptors, and is purely by co-operation or on a statistical basis. This multiple binding mimics nature (e.g. protein-protein \& protein-membrane binding), and results in significantly increased activity. Thus, attaching multiple copies of the ligand to dendritic surface promotes increased access to the target area, where the movement of the carrier system/ligand by simple diffusion is a problem. Nanoparticulate architecture of dendrimers favours its access in the highly permeable tumour vasculature and its high molecular weight causes its localisation and prevents its escape [99]. The process is termed the EPR effect.

- Dendrimers (MW>40 kDa) were found to remain in the blood for longer periods of time when compared with lower molecular weight polymers.

- The host-guest chemistry facilitates the encapsulation of hydrophobic drugs at the same time also stimulates the attachment of hydrophilic moieties.

- The hydrophilic exterior of these robust nanostructures prevents their recognition by mononuclear phagocytic system (MPS) and hence prevents their subsequent removal by opsonisation.

At the physiological $\mathrm{pH}(\sim 7.4)$ the tertiary amine groups of these dendrimers remain deprotonated and the branches converge to the central core. This prevents the release of drugs in the environment. However, once the dendrimers enter the tumour vasculature, which has a somewhat more acidic micro-environment, the amine groups protonate, and they repel to undergo a conformational change, thus promoting the release of drug.

\subsubsection{Receptor specific dendritic nanoconstructs}

The exo-groups that are at the surface of dendrimers can be designed so that few of the branches are conjugated to the drug, and the remaining ones are tailored to targeting moieties or ligands. Ligands attaching to the dendrimer confirm its destination to the target site and thus later prevent the delivery of the drug to non-target areas. Thus, polyvalency, i.e. the presence of multiple functional groups, aids with targeting the drug at its desired location. Spatial accessibility for targeting is provided by the presence of multiple branching sites in a dendrimer that provides enhanced interaction to the receptor. Targeted delivery offers increased therapeutic index, reduction in the required dose as well as toxicity. Dendrimers provide a unique platform that can couple the targeting moiety, drug, imaging agent and fluorescent probe simultaneously, without affecting the integrity of individual components. Dendrimers are different from various other carriers, which is better 
understood by the ability to attach any or all of these molecules in a well-defined and controllable manner onto a robust dendritic surface. This ability clearly differentiates dendrimers from other carriers such as micelles, liposomes, emulsion droplets, and engineered particles. Numerous tailorable surfaces on the dendrimer make it possible to attach various ligands and thus delivery to their specific receptors on the tumour cell surface or on the angiogenic microcapillaries growing around these cells. Specific cell surface receptors recognise structural analogues possessed by the ligands. Once the complex reaches the target site, it is internalised and subsequently releases the therapeutic moiety. The ligands thus play a prominent role in inhibiting or stimulating a patho-physiological response. Thus, conjugation of these ligands to dendrimers provides enhanced intracellular trafficking of these macromolecules in the necrotic tumour cells.

\subsubsection{Nanomedicine in the diagnosis and therapy of neurodegenerative disorders}

Alzheimer's and Parkinson's diseases i.e. neurodegenerative and infectious disorders, amyotrophic lateral sclerosis, and stroke are rapidly increasing as population's age. Early diagnosis would enable improved disease outcomes. Drugs, vaccines or regenerative proteins present "real" possibilities for positively affecting disease outcomes, but are limited in that their entry into the brain is commonly restricted across the blood-brain barrier (BBB). Such obstacles can be overcome by polymer science and nanotechnology which may improve diagnostic and therapeutic outcomes [100,101].

\subsubsection{Barriers to central nervous system (CNS) drug delivery [102]}

Formidable barriers that hinder delivery of diagnostic and therapeutic agents to CNS separates the brain from the rest of the body. Comprehending physiological features of these barriers is necessary for discovery of the means toward the effective delivery of drugs and imaging agents. Walls of capillaries in BBB separate the brain from circulating blood (Figure 7). 


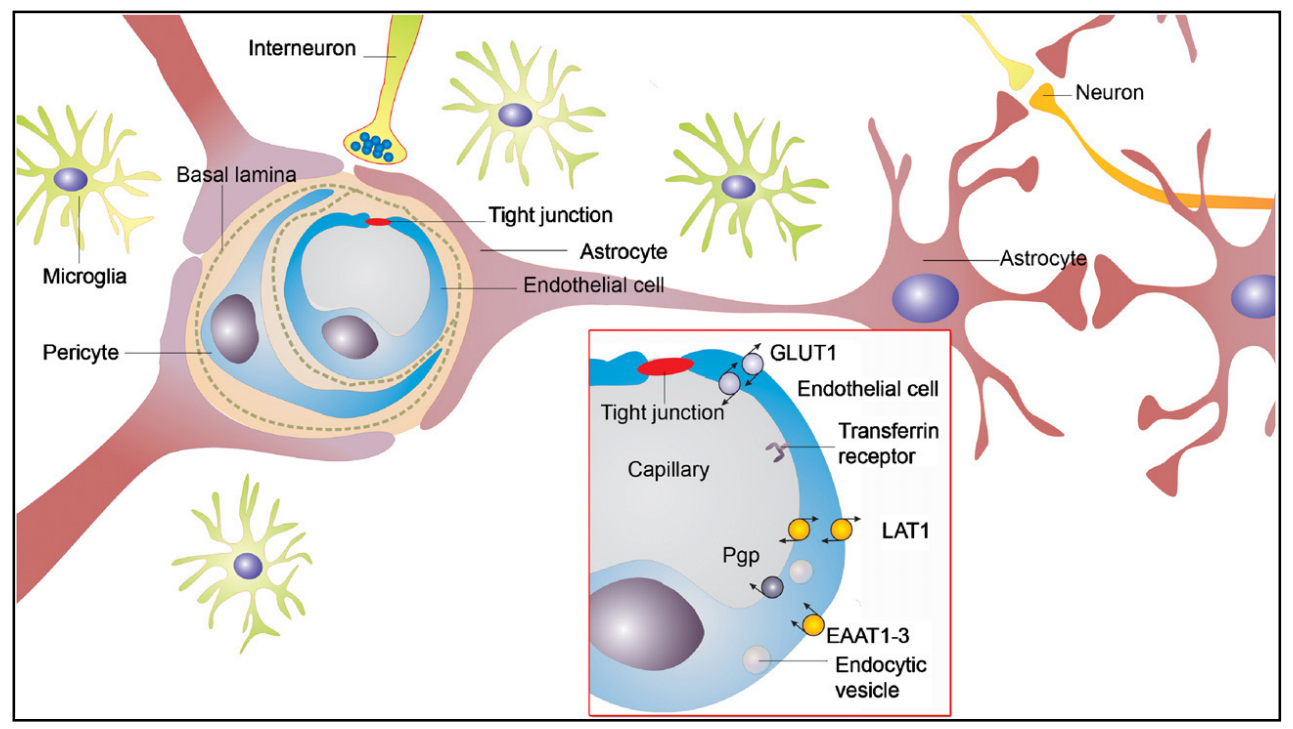

Figure 7. The BBB is formed by brain microvessel endothelial cells (BMVEC) that form tight junctions and express different transport systems such as Pgp, glucose transporter (GLUT1), large amino acid transporter (LAT1), excitatory amino acid transporters (EAAT1-3), transferrin receptor and others.

\subsubsection{Nanocarriers for CNS drug delivery [103]}

Nanocarriers are mainly low molecular weight drugs or therapeutic proteins that are chemically linked to water-soluble polymers. Linking to water soluble polymers increase drug solubility and drug stability, or enable the site-specific transport of drugs to target tissues affected by disease. Also, PEG-coated liposomes carrying chemotherapeutic drugs have been tested for clinical use.

\section{Liposomes}

Liposomes [104] are vesicular structures composed of unilamellar or multi-lamellar lipid bilayers surrounded by internal aqueous compartments.

Liposomes possess following advantages:

1. Varying sizes from several nanometres to several microns;

2. Large amounts of drug molecules can be incorporated into liposome aqueous compartments (water soluble compounds) or within lipid bilayers (lipophilic compounds) comparatively;

3. Reticuloendothelial system (RES) rapidly clears conventional liposomes from circulation; 
4. Extended circulation time can be accomplished with small sized liposomes $(\sim 10 \mathrm{~nm})$ composed of neutral, saturated phospholipids and cholesterol;

5. Liposomes with a surface modified with PEG ("PEGylation") reduces opsonisation of liposomes in plasma. Also it decreases its recognition and removal by the mononuclear phagocyte system (MPS) in liver and spleen;

6. PEGylated (or "stealth") liposomes have circulation half-life of around $50 \mathrm{~h}$ in humans. One such instance is that of doxorubicin encapsulated in PEGylated liposome, Doxils, which was approved for treatment of ovarian cancer, AIDS related Kaposi's sarcoma SS and metastatic breast cancer;

Encapsulation of a drug into liposomes prolongs drug circulation time in the blood stream, reduces drug side effects, and enhances drug therapeutic effects in CNS.

PEGylated liposomes $[105,106]$ coupled with monoclonal antibodies to glial fibrillary acidic protein (GFAP), an antigen expressed in astrocytes, show altered brain penetrance. Immunoliposomes are incapable of penetrating a normal BBB, while immunoliposomes used to treat glial brain tumours that express GFAP can reach their disease site when the BBB is partially permeabilised. Thus, the mechanism(s) of brain accumulation in disease may involve EPR of circulating liposomes at sites of disease-induced BBB compromise.

Alternatively, MP captures liposomes, which then cross the BBB. Liposomes have also been conjugated with mannose, transferrin and insulin receptors at the surface of brain capillaries. In particular, the transferrin receptor is necessary as it delivers iron across the BBB. The expression of this receptor in BBB increases during certain pathologies, for instance after stroke. Thus, transferrin-conjugated liposomes successfully targeted the post-ischemic brain endothelium in rats. Another example of a brain-targeting vector is a genetically engineered monoclonal antibody to human insulin receptor, 83-14 Mab. This vector was also used for targeting liposomes with the aid of a reporter gene to the brain.

\section{Nanoparticles}

Nanoparticles, used for drug and gene delivery are often composed of insoluble polymer(s). During their formulation, the drug is captured within the precipitating polymer, forming nanoparticles, and is then released upon the degradation of a polymer in the biological environment. The methods for the preparation of nanoparticles commonly employ the use of organic solvents, which may result in the degradation of immobilized drug agents, especially 
biomacromolecules. Efficient cell uptake is achieved when the nanoparticle size does not exceed 100-200 $\mathrm{nm}$.

Also, the nanoparticle surface is often modified by PEG that increases its dispersion stability and extends its circulation times in the body. For example, poly(butylcyanoacrylate) nanoparticles [107] were evaluated for the CNS delivery of many drugs. These nanoparticles were coated with PEG-containing surfactants, such as Tween 80. After injection, they localised in the choroid plexus, via mater and ventricles, and, to a lower extent, in the capillary endothelial cells. Also, some evidences suggest that increased brain delivery with surfactant-coated poly(butylcyanoacrylate) nanoparticles may be associated with non-specific permeabilisation of BBB and toxicity. Drugs delivered to CNS in these constructs included analgesics (Dalargin, Loperamide), anti-cancer agents (Doxorubicin), anti-convulsants (NMDA receptor antagonist, MRZ 2/576) and peptides.

More recently nanoparticles conjugated with metal chelators, Desferioxamine or D-Penicillamine, were shown to cross the BBB, chelate metals, and exit through the BBB with their complexed metal ions. This method may prove to be useful for reducing the metal load in neural tissue, thus later mitigating the harmful effects of oxidative damage during Alzheimer disease and other CNS diseases.

\section{Nanospheres}

Nanospheres are a subset of nanoparticles. Nanospheres [108] are hollow species prepared by microemulsion polymerisation or covering colloidal templates with a thin layer of polymer material followed by template removal. Such nanospheres remained carboxylated; polystyrene nanospheres $(20 \mathrm{~nm})$ were evaluated for CNS drug delivery in the vasculature under normal conditions after intravenous injection. However, they extravasated into the brain during cerebral ischemia-induced stress that partially opened the BBB. Such nanospheres may have the potential for imaging agents during ischemia, stroke and other conditions that disrupt the BBB and for the CNS delivery of drugs.

\section{Nanosuspensions}

Drug nanosuspensions [109] represent crystalline drug particles often stabilised by non-ionic PEG-containing surfactants or mixtures of lipids. They are manufactured by a variety of techniques such as media milling, high-pressure homogenisation or using emulsions and microemulsions as templates. These procedures often result in irregular shaped, rather polydisperse materials of near micron or sub-micron particle size range. Major advantages of this technology include its simplicity, high drug loading capacity and applicability to many drugs including very hydrophobic compounds. 
Nanosuspension surfaces can be modified to increase its delivery to the brain after systemic administration similar to that of regular nanoparticles.

Polymeric micelles

Polymeric micelles [110] ("micellar nanocontainers") have also been developed as carriers of drugs and diagnostic imaging agents. They form spontaneously in aqueous solutions of amphiphilic block copolymers [111]. They also have a core-shell architecture with a core of hydrophobic polymer blocks (e.g., poly(propylene glycol) (PPG), poly(D,L-lactide), poly(caprolactone), etc.) and a shell of hydrophilic polymer blocks (often PEG). The size of polymeric micelles usually varies from $10-100 \mathrm{~nm}$. Their core can incorporate considerable amounts (up to 20-30\% wt) of water-insoluble drugs that prevent premature drug release and degradation. The shell stabilises micelles in dispersion and masks the drug from interactions with serum proteins and untargeted cells. Diffusion helps in the release of the drug from the micelle after reaching the target cells (Figure 8).

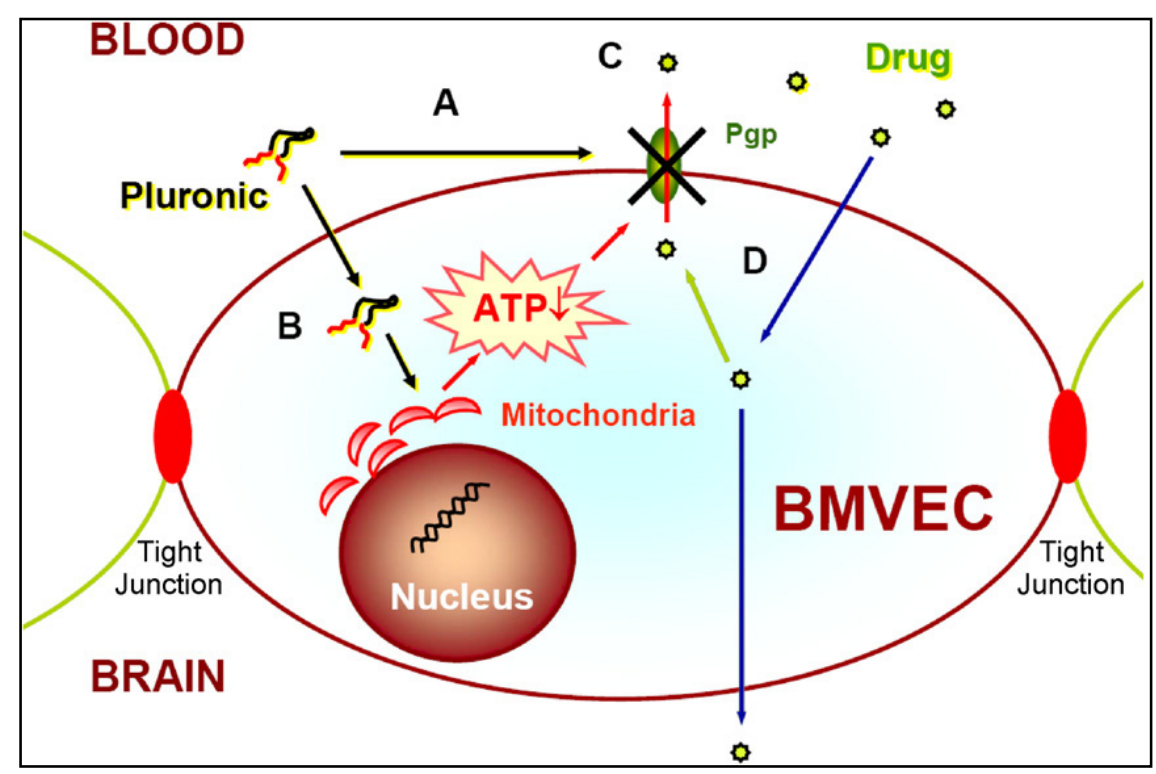

Figure 8. A scheme illustrating mechanism of Pluronics action in BBB: (A) inhibiting PgpATPase function in cell plasma membrane; (B) inhibiting respiration in mitochondria resulting in ATP depletion. Both effects combined result in (C) inhibition of the Pgp drug efflux system and (D) transport of the drug to the brain. 
For CNS drug delivery, an early study used micelles of Pluronic block copolymers [112] PEG-block-poly(propyleneglycol)-block-PEG (PEG-b-PPG-b-PEG) as carriers. These micelles were conjugated with either insulin as targeting moieties or polyclonal antibodies against brain a2-glycoprotein. Both antibody and insulin-vectorised micelles were shown to deliver a drug or fluorescent probe to the brain in vivo. Moreover, there was an increase in the neuroleptic activity of a drug (haloperidol) solubilised in the targeted micelles in comparison to a free drug.

Charged molecules can be incorporated by Polyion complex micelles [113] (also referred as "block ionomer complexes"), which are novel nanosystems. Formeation is a result of the reaction of double hydrophilic block copolymers, which contains ionic and non-ionic blocks along with macromolecules of opposite charge that includes oligonucleotides, plasmid DNA and proteins or surfactants of the opposite charge. For instance, by reacting trypsin or lysozyme (positively charged under physiological conditions) with an anionic block copolymer, block ionomer was made - PEG-poly(a,b-aspartic acid). These complexes spontaneously assemble into nanosized particles with core-shell architecture. The core has polyion complexes of a biomacromolecule and ionic block of the copolymer. Non-ionic block forms the shell. In the case of surfactant-based complexes, the core is composed of mutually neutralised surfactant ions and polyion chains. It contains hydrophobic domains of surfactant tail groups and has the ability to incorporate water-insoluble drugs. The complexes assume different morphologies that include vesicles and micelles of different shapes based on surfactant and block copolymer architectures.

These versatile nanomaterials can incorporate solutes of different structure with high loading capacity. Furthermore, they can release solutes upon changes in environmental conditions such as $\mathrm{pH}$ (acidification), concentration and chemical structure of elementary salt. DNA molecules in vitro and in vivo were efficiently delivered by nanomaterials.

Advances were made to develop stable polymeric micelles that do not dissociate during circulation in the body. Examples include amphiphilic scorpionlike block copolymers that have low critical micelle concentration (CMC) as well as various types of unimolecular micelles based on amphiphilic star-like macromolecules with covalently bound hydrophobic cores. Core-polymerisation was employed to stabilise micelles of heterotelechelic amphiphilic block copolymers which contained polymerisable groups at the ends of hydrophobic blocks. Wooley et al. have developed cage-like nanostructures on the base of polymeric micelles with hydrophobic core and cross-linked anionic shell. Additionally micelles with cross-linked ionic cores were prepared by self-assembly of ionic blocks of double hydrophilic block copolymers with a condensing agent, followed by the chemical cross-linking of ionic blocks [114]. The resulting micelles contain a hydrophilic PEO shell and a 
cross-linked hydrophilic ionic core, which is swollen in water and may incorporate hydrophilic drugs as well as imaging agents.

Protein and block ionomers were additionally cross linked with each other to improve the stability of polyion complex micelles with immobilised proteins according to another study. Similarly, in surfactant-based block ionomer complexes, surfactant molecules were chemically linked to each other ("dimerised"), which formed stable vesicles. Altogether, polymeric micelles of various types comprise a versatile platform for the delivery of imaging and therapeutic agents. One should expect further development of these systems for CNS drug delivery.

\section{Nanogels}

Nanogels are nanosized networks of cross-linked polymers that often combine ionic and non-ionic chains, such as PEI and PEG or poly(acrylic acid) and Pluronic [115]. Such networks swell in water and can incorporate through ionic interactions of oppositely charged molecules such as oligonucleotides, siRNA, DNA, proteins and low molecular mass drugs. Their loading proceeds with very high capacities (up to $40-60 \% \mathrm{wt}$ ) which are not achieved with conventional nanoparticles. Individual collapsed nanogel particles do not phase separate and form stable dispersions because of the solubility of PEG chains. Transport of oligonucleotides incorporated in nanogel particles across an in vitro model of the BBB was found satisfactory. It was because of the nanogels that the degradation of oligonucleotides decreased during their transport in brain microvessel endothelial cells (BMVEC). The surface of nanogels was modified by either transferrin or insulin, to further enhance delivery across the BBB. In vivo studies suggested that nanogel increased brain uptake of oligonucleotides while decreasing its uptake in the liver and spleen. To summarise, nanogels are promising carriers for CNS drug delivery, although they are in relatively early stages of development.

\section{Nanofibres and nanotubes}

Nanofibres and nanotubes [116] are carbon vapour grown, self-assembled from peptide amphiphiles or electrospun from most polymer materials. Carbon nanotubes have attracted attention in nanomedicine, even though there are also serious concerns regarding their safety. Electrospun continuous nanofibres are unique. It is because they represent nanostructures in two dimensions and macroscopic structures in another dimension. They are safer to manufacture than carbon nanotubes. They pose less of a risk of air pollution. Electrospun nanofibres of a degradable polymer, poly(lactic-co-glycolic acid) (PLGA) loaded with dexamethasone, have been used for neural prosthetic applications. A conducting polymer, poly(3,4-ethylenedioxythiophene), was deposited onto the nanofibre surface and the coated nanofibres were then mounted on the microfabricated neural microelectrodes, which were 
implanted into brain. Electrical stimulation released the drug that induced a local dilation of the coat and increased permeability. Nanotubes and nanofibres can be administered systemically in future, if the toxicity issues are addressed, for example, by appropriate polymer coating. Continuous nanofibres are more likely to be used in implants and also in tissue engineering applications.

\subsubsection{Nanoparticle applications in ocular gene therapy}

Nanoparticles can serve as carriers for drugs, peptides, vaccines and oligonucleotides and have been successfully delivered to multiple targets including cancerous cells, as well as other diseased tissues. Nanoparticles also have great potential as a strategy for gene therapy. They can be used to treat genetic defects in vitro and in vivo. Viral vectors have been the preferred mechanism for transfer of nucleic acids into tissues of interest historically, and they have dominated the field for some time. In a phase I/II clinical trial using a recombinant adeno-associated viral vector (rAAV) containing the herpes-simplex-virus thymidine kinase gene to treat hormone-refractory prostate cancer, two patients (out of six) responded positively to therapy [117].

Yet another instance of a successful viral gene therapy treatment comes from a phase I clinical trial using adeno-associated viral vector (AAV) to deliver pigment epithelium-derived factor (PEDF) to the eyes of patients diagnosed with age-related macular degeneration (AMD) and showed a significant level of reduction in neoangiogenesis associated with disease progression. Modified HIV vectors have been used to preserve some retinal function.

\section{Limitations of viral vectors [118]}

1. Physical limitations include random integration into the host's genome, immunogenicity of the vector, and limitations in the insert size;

2. Significant toxic side effects can be observed such as stimulation of an immune response, inflammation and neutralising antibodies, which are associated with repeat treatment, and other potentially serious toxic outcomes including death;

3. In addition of literature concerning the use of the most common AAV vectors for direct gene delivery is contradictory on both issues of transduction efficiency and inflammatory response and on the duration and reproducibility of transgene expression;

The lack of a clearly superior viral candidate for future clinical application of gene therapy in the eye combined with the limitations of viral gene therapy mentioned above make the development of an efficacious non-viral vector for the eye of supreme importance.

Liposomes, DNA nanoparticles or combination of both are included into non-viral vectors. Although liposomes are promising, they have shown low transfection efficiency. This can cause significant inflammatory toxicity. 
Alternatively, compacted DNA nanoparticles have proven to be a very useful vehicle for gene therapy and meet the majority of the requirements discussed above for a successful vector [119]. There are many different formulations of nanoparticles. They typically contain a segment of DNA or RNA (circular or linear) which is compacted with a polycationic polymer. Their size is quite small, typically ranging from $10-100 \mathrm{~nm}$ in diameter. These small particles are taken up at the cell surface and trafficked to the nucleus within a short period of time. The delivery of compacted DNA nanoparticles to the target yields medium to high transfection efficiency; in many cases, expression levels are several-fold greater than those observed after treatment with naked plasmid DNA. These results are dependent on specifics of the nanoparticle formulation, size, or electric charge. Excellent preliminary studies have been undertaken with poly(lactic acid) and poly(lactide co-glycolide) nanoparticles in the retina, and until now far they have not been used for gene transfer to the mammalian retina. Compacted PEG nanoparticles have been used to efficiently transfect post-mitotic cells in vitro and in vivo.

\section{Advantages of non-viral vectors}

1. These nanoparticles can be stably stored under a variety of conditions and concentrations (up to $12 \mathrm{mg} \mathrm{ml}^{-1}$ of DNA);

2. They are tolerant of a wide range of temperatures, salt concentrations and $\mathrm{pH}$;

3. DNA or RNA is protected from DNase or RNase degradation that is a tendency of non-viral vectors;

4. One of the most exciting features of compacted DNA/RNA nanoparticles is their insert capacity. Some DNA-compacted nanoparticles can contain plasmids up to $20 \mathrm{~kb}$ and retain full functional competence following in vivo administration;

5. Studies in humans and mice showed little to no toxicity in the targeted tissues. Also, they showed modest immune response when a high concentration of the nanoparticles was used;

6. No serious side effects were observed even after repetitive administration of nanoparticles is possible.

\section{Limitations of non-viral vectors}

1. Low transfection efficiency;

2. Short duration of action and expression;

3. One of the traditional limitations of non-viral vectors has been passage of the vector across two physiological barriers: the cell membrane and the nuclear membrane. Endocytosis is the most commonly accepted pathway for nanoparticle internalisation into the cytosol. Endocytosis 
can be divided into two categories, phagocytosis (which requires specialised cells) or pinocytosis. The latter pathway can be subdivided into macropinocytosis (molecules $>120 \mathrm{~nm}$ ), caveolin-mediated endocytosis (molecules $60 \mathrm{~nm}$ ) and clathrin-mediated endocytosis $(120 \mathrm{~nm})$. Positively charged nanoparticles use clathrin-mediated endocytosis.

To summarise, compacted-DNA nanoparticle-mediated gene therapy provides a safe, effective and promising system for the delivery of therapeutic genes to target tissues in the eye. They drive very specific and high levels of gene expression and expression that can be sustained for several months. The safe use of compacted DNA nanoparticles in the clinical setting speaks to their viability as a potential treatment strategy for human conditions. The use of this system in the treatment of genetic diseases of the eye is a strong alternative to the existing collection of viral vectors.

\subsubsection{Nanoparticles for the treatment of osteoporosis}

There are no current effective prevention and treatment methods for this disease; even though the studies have been taking place since years. There are several major barriers that exist for the use of any pharmaceutical agents to stimulate new bone formation. First, the agents can cause non-specific bone formation in undesirable areas. This is because these agents are often delivered in non-specific ways (such as through the mouth, directly into the blood stream, etc.). Second, if delivered locally to the tissue around the area of low bone density, they rapidly diffuse to adjacent tissues. This limits their potential to promote prolonged bone formation in targeted areas of weak osteoporotic bone. Even the best strategies to sufficiently increase bone mass (although, to date, still unproven) require at least one year to see any change; for these reasons, this is a time period that is not acceptable, especially for the elderly. Thus for these reasons, nanotechnology will be used (or the design of materials with $10^{-9} \mathrm{~m}$ dimensions) to develop novel drug-carrying systems that will specifically attach to osteoporotic (not healthy) bones. Moreover, some of these novel drug carrying systems will then distribute pharmaceutical agents locally in order to quickly increase bone mass.

Nanoparticles offer a high potential for several biomedical applications, including bioanalysis and bioseparation, tissue-specific drug therapeutic applications, gene and radionuclide delivery are based on their unique mesoscopic physical, chemical, thermal, and mechanical properties. To be used effectively in fighting diseases, specific surface chemistry of the nanoparticles need to be tailored in order to have desired biomedical applications. Magnetic nanoparticles are also of interest [120]. Specifically, the main interest in the use of magnetic nanoparticles in biomedical applications is that a homogeneous external magnetic field exerts a force on them, and this is how they can be manipulated or transported to a specific diseased tissue by a 
magnetic field gradient. They also have controllable sizes to match their dimensions either that of a virus $(20-500 \mathrm{~nm})$, of a protein $(5-50 \mathrm{~nm})$ or of a gene ( $2 \mathrm{~nm}$ wide and 10-100 nm long). After removal of the magnetic field, magnetic particles are of interest because they do not retain any magnetism.

Nanotechnology is specifically used here to prolong the release of bioactive agents that efficiently regenerate enough bone for a patient to return to a normal active lifestyle. To be specific, inorganic biodegradable nanoparticles (including ceramics like hydroxyapatite) will be functionalised with bioactive chemicals such as bone morphogenetic protein-2 (BMP-2) that bond to bone of low mass. Bioactive groups like these will be placed on the outer surface of the magnetic nanoparticle systems using various techniques (such as covalent chemical attachment). Once bonded specifically to osteoporotic bone and not healthy bone, magnetic nanoparticle systems will deliver bioactive compounds to locally increase bone mass. Lastly, the outer coating of the embedded nanoparticle systems will be created to have different biodegradation rates for the release of bone-building agents over various time spans. This allows for not only quick bone formation, but also long-term sustained bone regeneration. One potential advantage of formulating hyaluronan (HA) magnetic nanoparticles is that, as the magnetic particles accumulate, e.g., in bone tissue, they can play an important role in detection through MRI to locate, monitor and control drug activities.

\subsubsection{Applications of nanotechnology in diabetes}

Diabetes mellitus, often referred to simply as diabetes, is a chronic metabolic disorder due to the relative deficiency of insulin secretion and varying degrees of insulin resistance. It is characterised by high circulating glucose. Excessive levels of either molecular oxygen or Reactive Oxygen Species (ROS) lead to an imbalance in the body's normal oxidative metabolism that to leads to high glucose levels in the blood (hyperglycemia), resulting in metabolic disturbances (oxidative stress) and chronic complications in diabetes. The management of diabetic conditions by insulin therapy has several drawbacks like insulin resistance. Also, chronic treatment causes anorexia nervosa, brain atrophy and fatty liver. Several research studies are currently ongoing with the aid of nanosize particles to overcome such limitations in diabetes management [121,122].

\subsubsection{Nanomedicine application in glucose and insulin monitoring}

The major problems with conventional finger-prick capillary blood glucose self-monitoring are widely accepted [123]. It is painful (leading to non-compliance) and cannot be performed when the patient is sleeping or driving a motor vehicle (times when the patient is especially vulnerable to hypoglycemia). Since it is intermittent, it can miss dangerous fluctuations in blood glucose concentrations between tests. A new method that uses 
nanotechnology to rapidly measure minute amounts of insulin and blood sugar level is a major step toward developing the ability to assess the health of the body's insulin-producing cells.

\section{Microphysiometer}

The microphysiometer is built from multiwalled carbon nanotubes, which are like several flat sheets of carbon atoms stacked rolled into very small tubes. It can be used to detect and monitor the response of cells to a variety of chemical substances, especially ligands for specific plasma membrane receptors. The nanotubes are electrically conductive. The concentration of insulin in the chamber can be directly related to the current at the electrode. The nanotubes operate reliably at $\mathrm{pH}$ levels characteristic of living cells. Current detection methods measure insulin production at intervals by periodically collecting small samples and then later measuring their insulin levels. The new sensor detects insulin levels continuously. This is possible by measuring the transfer of electrons produced when insulin molecules oxidise in the presence of glucose. When the cells produce more insulin molecules, the current in the sensor increases or vice versa, allowing insulin concentrations to be monitored in real-time [124].

\section{Implantable sensor}

An implantable sensor capable of long-term monitoring of tissue glucose concentrations by wireless telemetry has been developed for eventual application in people with diabetes. The implantable sensor is designed to give diabetes patients an alternative to finger-sticking or short-term glucose sensors. It also limit dangerous glucose level fluctuations known as glucose excursions. The use of PEG beads coated with fluorescent molecules to monitor diabetes blood sugar levels is very effective. In this method, the beads are injected under the skin and stay in the interstitial fluid. Glucose displaces the fluorescent molecules and creates a glow when glucose in the interstitial fluid drops to dangerous levels. This glow is seen on a tattoo placed on the arm. Sensor microchips are also being developed to continuously monitor key body parameters including pulse, temperature and blood glucose. A chip implanted under the skin would transmit a signal that could be monitored continuously [125].

\subsubsection{Nanoparticles in the treatment of diabetes}

\section{Polymeric nanoparticles}

Polymeric nanoparticles have been used as carriers of insulin and the use of biodegradable polymeric nanoparticles for controlled drug delivery has shown significant therapeutic potential. These are biodegradable polymers that are with the polymer-insulin matrix surrounded by the nanoporous membrane 
containing grafted glucose oxidase. A rise in blood glucose level triggers a change in the surrounding nanoporous membrane that results in biodegradation and subsequent insulin delivery. Lowering of the $\mathrm{pH}$ in the delivery system's microenvironment is due to the glucose/glucose oxidase reaction. This may cause an increase in the swelling of the polymer system, leading to an increased release of insulin. $N, N$-dimethylaminoethyl methacrylate and poly(acrylamide) are the polymers investigated for such applications. This "molecular gate" system is composed of an insulin reservoir and a delivery ratecontrolling membrane which is made of poly[methacrylic acid-g-PEG] copolymer. The polymer swells in size at normal body $\mathrm{pH}$ $(\mathrm{pH}=7.4)$ and closes the gates. It shrinks at low $\mathrm{pH}(\mathrm{pH}=4)$ when the blood glucose level increases, thus opening the gates and releasing the insulin from the nanoparticles. These systems release insulin by swelling which is caused due to changes in blood $\mathrm{pH}$. The control of the insulin delivery depends on the size of the gates, the concentration of insulin, and the rate of gates' opening or closing (response rate). These self-contained polymeric delivery systems are still under research. Now, the delivery of oral insulin with polymeric nanoparticles has progressed to a greater extent in the recent year [126].

\section{Oral insulin administration by using polysaccharides} and polymeric nanoparticles

Polysaccharides are natural biodegradable hydrophilic polymers. These exhibit enzymatic degradation behaviour and good biocompatibility. The development of improved oral insulin administration is essential for the treatment of diabetes mellitus in order to overcome the problem of daily subcutaneous injections. Insulin undergoes degradation in the stomach due to gastric enzymes when administered orally; therefore, insulin should be enveloped in a matrix-like system to protect it from gastric enzymes. This can be achieved by encapsulating the insulin molecules in polymeric nanoparticles. Calcium phosphate-PEG-insulin combination was combined with casein (a milk protein) in one such study. The insulin is protected from the gastric enzymes by the casein coating. Due to casein's mucoadhesive property, the formulation remained concentrated in the small intestine for a longer period, resulting in slower absorption and longer availability in the bloodstream [127].

\section{Insulin delivery through inhalable nanoparticles}

Inhalable, polymeric nanoparticle-based drug delivery systems have been tried previously for the treatment of tuberculosis. Such approaches can be directed toward insulin delivery through inhalable nanoparticles. Insulin molecules can be encapsulated within the nanoparticles and can be administered into the lungs by inhaling the dry powder formulation of insulin. The nanoparticles should be small enough to avoid clogging up the lungs but large enough to avoid being exhaled. Such a method of administration allows the direct delivery of insulin molecules to the bloodstream without undergoing 
degradation. A few studies have been performed to test the potential use of ceramic nanoparticles (calcium phosphate) as drug delivery agents. Porous hydroxyapatite nanoparticles have also been tested for the intestinal delivery of insulin. Preclinical studies in guinea pig lungs with insulin-loaded poly(lactide-co-glycolide) nanospheres demonstrated a significant reduction in blood glucose level with a prolonged effect over $48 \mathrm{~h}$ when compared with insulin solution. Insulin-loaded poly(butyl cyanoacrylate) nanoparticles when delivered to the lungs of rats were shown to extend the duration of hypoglycaemic effect over $20 \mathrm{~h}$ when compared with the pulmonary administration of insulin solution. The major factors limiting the bioavailability of nasally administered insulin include poor permeability across the mucosal membrane and rapid mucociliary clearance mechanism which removes the non-mucoadhesive formulations from the absorption site. Mucoadhesive nanoparticles made of chitosan/tripolyphosphate and starches have been evaluated to overcome these limitations. These nanoparticles showed good insulin-loading capacity. It provided the release of $75 \%$ to $80 \%$ insulin within 15 min after administration [123].

\subsubsection{Applications of nanotechnology in diabetes}

Diabetes is one of the major afflictions of modern western society. To date, diabetic patients control their blood-sugar levels via insulin introduced directly into the blood stream using injections. This unpleasant method is required since stomach acid destroys protein-based substances including insulin, making oral insulin consumption useless. The new system is based on inhaling the insulin (instead of injecting it) and on the controlled release of insulin into the bloodstream (instead of manually controlling the amount of insulin injected). The treatment of diabetes includes the proper delivery of insulin into the blood stream, which can be achieved by nanotechnology in the following ways:

\section{Development of oral insulin}

The production of pharmaceutically active peptides and proteins, such as insulin, in large quantities has become feasible. The oral route is considered to be the most convenient and comfortable means for the administration of insulin for less invasive and painless diabetes management. This leads to higher patient compliance. As hydrophilic drugs cannot diffuse across epithelial cells through the lipid bilayer cell membranes into the bloodstream, the intestinal epithelium is a major barrier to the absorption. Therefore, attention has been given to improving the paracellular transport of hydrophilic drugs. A variety of intestinal permeation enhancers including chitosan have been used for the assistance of the absorption of hydrophilic macromolecules. Hence, a carrier system is needed to protect protein drugs from the harsh environment in the stomach and small intestine, if given orally. Additionally, chitosan nanoparticles enhanced the intestinal absorption of protein molecules 
to a greater extent than aqueous solutions of chitosan in vivo. The insulin loaded nanoparticles coated with mucoadhesive chitosan may prolong their residence in the small intestine, infiltrate into the mucus layer and subsequently mediate transiently opening the tight junctions between epithelial cells while becoming unstable and broken apart due to their $\mathrm{pH}$ sensitivity and/or degradability. The insulin released from the broken-apart nanoparticles could then permeate through the paracellular pathway to the bloodstream, which is its ultimate destination [128].

\section{Microsphere for oral insulin production}

The most promising strategy to achieve oral insulin is the use of a microsphere system. It is inherently a combination strategy. The oral drug delivery device for insulin is used to protect the sensitive drug from digestive enzymes and proteolytic degradation in stomach and upper part of gastrointestinal tract. Microspheres act as protease inhibitors by protecting the encapsulated insulin from enzymatic degradation within its matrix as well as permeation enhancers by effectively crossing the epithelial layer after oral administration [129].

\section{Artificial pancreas}

An artificial pancreas system is an automated, closed-loop system that combines a continuous glucose monitor, an insulin infusion pump, and a glucose meter for calibrating the monitor. The devices are designed to work together, monitor the body's glucose levels and automatically pump appropriate doses of insulin as determined by a computer algorithm [89]. The development of an artificial pancreas could be the permanent solution for diabetic patients. The concept of its work is simple: a sensor electrode repeatedly measures the level of blood glucose; this information feeds into a small computer that energises an infusion pump, and the needed units of insulin enter the blood stream from a small reservoir. Another way to restore body glucose is the use of a tiny silicon box containing pancreatic beta cells taken from animals. The box is surrounded by a material with a very specific nanopore size (about $20 \mathrm{~nm}$ in diameter). These pores are big enough to allow for glucose and insulin to pass through them, but small enough to impede the passage of much larger immune system molecules. These boxes can be implanted in diabetes patients under their skin. This could temporarily restore the body's delicate glucose control feedback loop without the need for a powerful immunosuppressant which may leave the patient at a serious risk of infection. Scientists are also trying to create a nanorobot which would have insulin departed in inner chambers, and glucose level sensors on the surface. When blood glucose levels increase, the sensors on the surface would record it and insulin would be released. Yet, this kind of nano-artificial pancreas is still only a theory. In the artificial pancreas, biosensors are also useful [130]. 


\section{The nanopump}

The nanopump is a powerful device with many possible applications in the medical field. The first major application of the pump, introduced by Debiotech, was insulin delivery. The pump injects insulin to the patient's body at a constant rate, balancing the amount of sugars in his or her blood. The pump can also administer small drug doses over a long period of time.

\subsubsection{Nanosystems in inflammation}

\subsubsection{Targeting macrophages to control inflammation}

The potential of macrophages for rapid recognition and clearance of foreign particles has provided a rational approach to macrophage-specific targeting with nanoparticles. Macrophages' have the ability to secrete a multitude of inflammatory mediators that allows them to regulate inflammation in many diseases. Therefore, macrophages are potential pharmaceutical targets in various human and animal diseases. Although macrophages are capable of killing most of the microbes, many microorganisms (Toxoplasma gondii, Leishmania sp, Mycobacterium tuberculosis, and Listeria monocytogenes) have developed the potential ability to resist the phagocytosis activity of macrophages. These pathogens subvert a macrophage's molecular machinery designed to kill them and come to reside in modified lysosomes. Therefore, nanoparticle-mediated delivery of antimicrobial agent(s) into pathogencontaining intracellular vacuoles in macrophages could be useful and help to eliminate cellular reservoirs [131]. This system can be used to achieve therapeutic drug concentrations in the vacuoles of infected macrophages. It is also used in the reduction of side effects associated with the drug administration and the release of pro-inflammatory cytokines. Poly(alkyl cyanoacrylates) (PACAs) nanoparticles have been used as a carrier for targeting anti-leishmanial drugs into macrophages. This nanomaterial did not induce interleukin-1 release by macrophages [132]. Therefore, in chronic diseases, similarly designed nanosytems could be very useful in targeting macrophage infections.

The antifungal and anti-leishmanial agent amphotericin B (AmB) has been complexed with lipids-based nanotubes in order to develop a less toxic formulation of AmB. Gupta and Viyas [133] formulated AmB in trilaurin based nanosize lipid particles (emulsomes) stabilised by soya phosphatidylcholine as a new intravenous drug delivery system for macrophage targeting. Nanocarrier-mediated delivery of macrophage toxins has proved to be a powerful approach in getting rid of unwanted macrophages in gene therapy. Also nanocarrier-mediated delivery of macrophage toxins is useful in other clinically relevant situations such as autoimmune blood disorders, $\mathrm{T}$ cell-mediated autoimmune diabetes, rheumatoid arthritis, spinal cord injury, sciatic nerve injury, and restenosis after angioplasty. Alternatively, 
nanoparticles with macrophage-lethal properties can also be exploited. Exploiting a variety of macrophage cell receptors as therapeutic targets may prove a better strategy for antigen delivery as well as targeting with particulate nanocarriers.

\subsubsection{Targeting inflammatory molecules}

Many cell adhesion molecules have been discovered in the past two decades. Cell adhesion molecules are glycoproteins found on the cell surface which act as receptors for cell-to-cell and cell-to-extracellular matrix adhesion [134]. These cell adhesion molecules are divided into four classes called integrins, cadherins, selectins, and the immunoglobulin superfamily. These molecules are required for the efficient migration of inflammatory cells such as neutrophils and monocytes into inflamed organs and the generation of host response to infections. However, there is considerable evidence that excessive migration of neutrophils in inflamed lungs leads to exaggerated tissue damage and mortality. Therefore, a major effort is underway to fine tune the migration of neutrophils into inflamed organs. Recent advancements of the understanding of the cell adhesion molecules has impacted the design and development of drugs (i.e. peptide, proteins) basically for the potential treatment of cancer, heart and autoimmune diseases $[135,136]$. These molecules have important roles in diseases such as cancer [137], thrombosis and autoimmune diseases like type- 1 diabetes. The arginylglycylaspartic acid (RGD) peptides have been used to target integrins $\alpha v \beta 3$ and $\alpha v \beta 5$, and peptides derived from the intercellular adhesion molecule-1 (ICAM-1) have been used to target the $\alpha v \beta 2$ integrin. Peptides derived from $\alpha v \beta 2$ can target ICAM- 1 expressing cells. Cyclic RGD peptides have been conjugated to paclitaxel (PTX-RGD) and doxorubicin (Dox-RGD4C) for improving the specific delivery of these drugs to tumour cells. Mice bearing human breast carcinoma cells (i.e., MDA-MB-435) survived the disease when treated with Dox-RGD4C, while all of the untreated control mice died because of the disease [138]. $\alpha v \beta 3$ and $\alpha v \beta 5$ integrins on the tumour vasculature during angiogenesis are targeted by this conjugate.

Extracellular regulated kinases (ERK) may regulate apoptosis and cell survival at multiple points that include increasing p53 and BAX action, increasing caspase- 3 and caspase- 8 activities, decreasing Akt activity, and increasing the expression of tumor necrosis factor alpha (TNF- $\alpha$ ) [139]. Our research group is investigating the interaction of RGD-Rosette nanotubes (RGD-RNT) to $\alpha v \beta 3$ integrins, following cell signalling through P38 kinases and its function in human lung epithelial cells, and bovine and equine neutrophil migration. Cyclo(1,12)PenITDGEATDSGC peptide (cLABL peptide), derived from the I-domain of the $\alpha$ subunit of leukocyte function-associated factor-1 (LFA-1), is known to bind ICAM-1. The cLABL peptide has been conjugated with methotrexate (MTX) to give the MTX-cLABL conjugate. Because ICAM-1 is upregulated during tissue inflammation and several different cancers, this conjugate may be useful for directing drugs to inflammatory and tumour cells. 
The anti-inflammatory activity of MTX is due to the suppression of the production of anti-inflammatory cytokines such as (interleukin-6) IL-6 and (interleukin-8) IL-8. Thus, the activity of MTX-cLABL conjugate was compared to MTX in suppressing the production of these cytokines in human coronary artery endothelial cells stimulated with TNF- $\alpha$. MTX-cLABL is more selective in suppressing the production of IL-6 than IL-8, which is opposite to MTX. PLGA nanoparticles coated with cLABL peptides have also been shown to upregulate ICAM-1 [140]. More detailed information on the mechanism(s) of internal isolation and intracellular trafficking of cell adhesion molecules is required to be exploited for delivering drug molecules to a specific cell type or for the diagnosis of cancer and other diseases (heart and autoimmune diseases).

\subsubsection{Nanotechnology in hypertension [141]}

For the flow of blood through arteries, it requires some force, which is measured in terms of blood pressure. When the flow of blood changes, blood pressure may elevate or lower according to the flow. When blood pressure is elevated, the heart has to work harder than normal so that the proper flow of blood through blood vessels is possible. The blood pressure in the arteries is elevated; this phenomenon is known as hypertension, high blood pressure or arterial hypertension. It is a chronic disease. Blood pressure at rest is within the range of 100-140 $\mathrm{mm} \mathrm{Hg}$ systolic (top reading) and 60-90 $\mathrm{mm} \mathrm{Hg}$ diastolic (bottom reading) in normal conditions. If the blood pressure is at or above $140 / 90 \mathrm{~mm} \mathrm{Hg}$, it is termed high blood pressure. A blood pressure greater than $180 / 110 \mathrm{~mm} \mathrm{Hg}$ is termed a 'hypertensive crisis.' Headaches, light-headedness, vertigo, tinnitus, and altered vision are the symptoms of hypertension. Physical examination can be performed by examining optic fundus in the back of the eye for the presence of hypertensive retinopathy.

\subsubsection{Cause}

Hypertension is classified as either primary hypertension or secondary hypertension. Primary hypertension is caused by high salt intake, high alcohol consumption, and the use of high fat products. Also, stress plays a minor role. For adult primary hypertension, early life events like low birth weight, maternal smoking, lack of breast feeding, etc. can be implicated as risk factors. Some risk factors for secondary hypertension are endocrine conditions, sleep apnoea, obesity, excessive liquorice, illegal drugs, and herbal medicines. Hypertension may result from a complex interaction of genes and environmental factors.

\subsubsection{Diagnosis and drugs}

The diagnosis of hypertension is made on the basis of a persistently high blood pressure. Some typical tests performed in hypertension are given in Table 2. 
Table 2. The tests used in the diagnosis of hypertension

\begin{tabular}{cr}
\hline System & Tests \\
\hline Renal & Microscopic urinalysis, Proteinuria, BUN, Creatinine \\
\hline Endocrine & Serum sodium, calcium, potassium, TSH \\
\hline Metabolic & Fasting blood glucose, HDL, LDL, Total cholesterol, triglycerides \\
\hline Others & Haematocrit, Chest radiograph, Electrocardiogram \\
\hline & Source: Harrison's principles of internal medicine
\end{tabular}

For the treatment of hypertension, a health care provider (Doctor) will advise to lose weight, stop smoking and start exercising. Some medicines for high blood pressure are:

$>$ Diuretics - These pills help the kidneys to remove salt from blood, e.g. Chlorthalidone, Hydrochlorothiazide.

$>$ Beta blockers - These pills will help heart beat slow and lower the pressure.

$>$ Angiotensin-converting enzyme inhibitors (ACE Inhibitors) - These inhibitors make the blood vessels relax, so that the blood pressure lowers.

$>$ Angiotensin II receptor blockers- These blockers work the same as ACE Inhibitors, e.g. Olmesartan, Medoxomil.

$>$ Calcium channel blockers - These blockers stop calcium from entering the cells that will relax blood vessels.

$>$ Renin Inhibitors - These newer types of medicines act by relaxing blood vessels to control blood pressure.

$>$ Sometimes, patients have to take more than one drug/medicine to control blood pressure. These medicines may have several side effects such as:

- Cough, Diarrhoea

- Erection problems

- Feeling tired, weak, nervous

- Skin rash

- Weight loss or gain without trying 


\subsubsection{Nanoparticle based hypertension treatment}

In the pulmonary artery, when pulmonary vascular resistance increases and the right ventricle fails, this is known as pulmonary arterial hypertension (PAH). In the treatment of $\mathrm{PAH}$, currently the most effective drugs have a very short life-cycle, difficult rendering systemic administration. As a future prospective, these difficulties can be overcome by using nanotechnology.

$\mathrm{PAH}$, asthma and chronic obstructive pulmonary disease (COPD) share pathological features, such as inflammation and smooth muscle contraction. No existing drugs have the potential to deal with these pathomechanisms. Using a combination of long active vasoactive intestinal peptide (VIP) analogues with drug delivery systems can provide clinically useful agents for the treatment of pulmonary hypertension in asthma and COPD. Nanoparticles used as drug delivery systems:

Liposomes - Liposomes are concentric nanosized artificial vesicles with a spherical shape that can be produced from natural phospholipids and cholesterol. Liposomes size should be big enough to carry a sufficient amount of drugs. After the encapsulation of drugs, liposomes should protect their payload from degradation in the microenvironment of the pulmonary artery and a controlled, retarded release of the drugs.

Micelles - Self-assembled supermolecular structures consisting of amphiphilic macromolecules are micelles. When coming into contact with water, these amphiphilic molecules assemble to form ananoscopic core-shell structure that can be used as a reservoir for hydrophobic drugs. PEG-based cationic micelles are used for intratracheal gene transfer to the lung of rats with monocrotaline-induced PAH. A remarkable therapeutic efficiency was achieved without compromising biocompatibility using these nanoparticles.

Polymeric nanoparticles - From previous studies of microspheres and submicron particles, the use of polymeric nanoparticles was derived. Generating aqueous droplets in the range of $1-3 \mu \mathrm{m}$ provides the chance to carry numerous nanoparticles in one droplet. The feasibility of polymeric microspheres as an inhalable carrier for prostaglandin E1 (PGE1) for the treatment of PAH was studied using PLGA microspheres. Another approach for the treatment of PAH is using combination of PEO- $w$-lactic acid (PELA) and hydrophilic prodrug (PROLI/NO) has not yet been successful, even using nanoparticles. Further studies are continuing for the success of this method.

In another approach, bioabsorbable polymeric nanoparticles formulated from a PEG-PLGA enabled the delivery of the nuclear factor $\kappa \mathrm{B}(\mathrm{NF}-\kappa \mathrm{B})$ decoy oligodeoxynucleotide, which is directed against NF- $\kappa \mathrm{B}$ binding site in the promoter region. This study in a rat model of monocrotaline-induced PAH showed that these nanoparticles prevent monocrotaline-induced NF- $\kappa \mathrm{B}$ activation. 
Nanocrystals and nanoprecipitates - Nifedipine (hypertension drug) nanoparticles are co-precipitated with steric acid to form a negative surface charged colloid. Destabilisation of the colloid was performed by using $\mathrm{NaCl}$ to disrupt the electrostatic repulsion between the particles in order to achieve agglomerated nanoparticles of a controlled size. Such nanoparticles are well suited for pulmonary delivery. Tranilast, an anti-allergic agent that has a potential for the co-treatment of pulmonary inflammation during PAH, can be processed as wet-milled crystalline particle that possess a mean diameter of $122 \mathrm{~nm}$.

\subsubsection{Biomedical nanotechnology}

Three applications of nanotechnology are particularly suited to biomedicine, namely diagnostic techniques, drugs and prostheses and implants [142]. Interest is booming in biomedical applications for use outside the body, like diagnostic sensors and "lab-on-a-chip" techniques that are suitable for analysing blood and other samples, and for inclusion in analytical instruments for research and development (R\&D) on new drugs. Many companies are developing nanotechnology applications for anticancer drugs, implanted insulin pumps, and gene therapy for inside the body. Many other researchers are working on prostheses and implants which include nanostructured materials.

The applications include:

1. Sensors needed for medical and environmental monitoring and for preparing pure chemicals and pharmaceuticals (Figure 9).

2. Light and strong materials for defence, aerospace, automotive and medical applications.

3. Lab-on-a-chip diagnostic techniques.

4. Sunscreens with ultraviolet-light absorbing nanoparticles.

5. The report also said that the following applications are expected in the next decade:

6. Longer-lasting medical implants.

7. The capability to map an individual's entire genetic code almost instantaneously.

8. The ability to extend life by $50 \%$ from present expectations. 


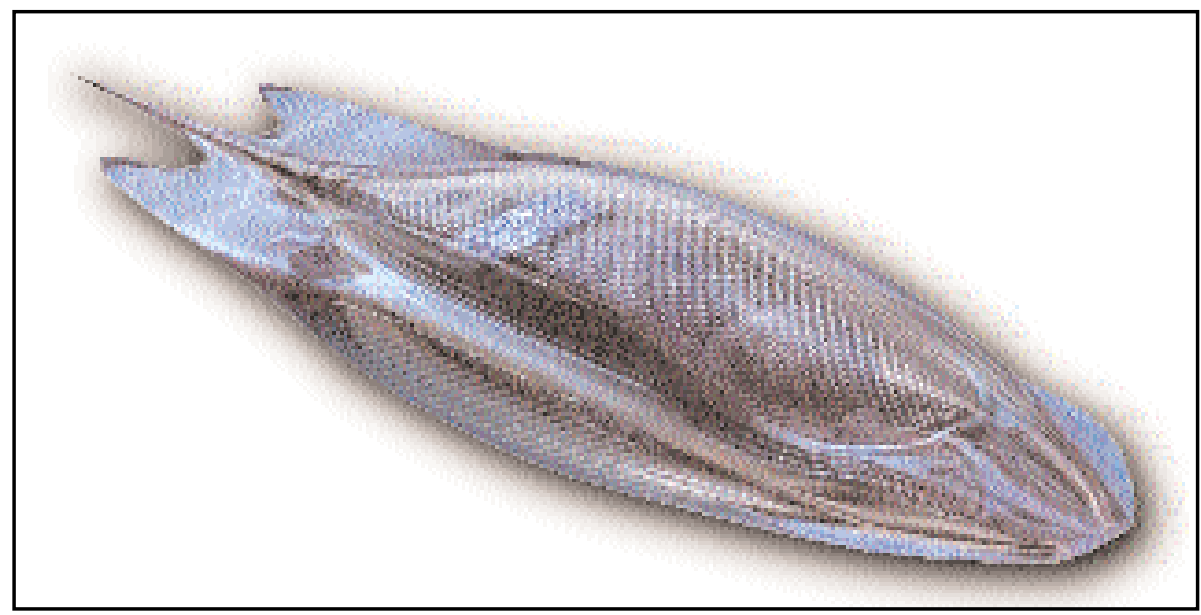

Figure 9. A bioengineered cell rover swims through the human body in this artist's impression, performing drug delivery, waste removal, and cellular repair. It has an internal frame, skin panels, internal organelles, a propulsion scheme, and a control system.

\section{Nanodrugs}

Nanostructured materials becoming new drug compounds are not expected by the pharmaceutical companies. However, carbon buckyballs and nanotubes might be useful as drug delivery vehicles because their nanometre size enables them to move easily inside the body. The active compound might be inserted in a nanotube or bonded to a particle's surface. Also, other types of nanopowders or biomolecules are also useful. For example, albumin-bound paclitaxel (ABI-007) is a new nanoparticle delivery system for an established anticancer drug. ABI-007 is $130 \mathrm{~nm}$ long and consists of an engineered protein-stabilised nanoparticle that contains paclitaxel, which is used to treat breast, bladder, and more than a dozen other cancers. New delivery systems like these combine a drug with an artificial vector that can enter the body and move in it like a virus.

Cosmetics based on QDs are already sold in large quantities. Nanophase Technologies Corp. (Romeoville, IL) produces nanocrystalline materials like zinc oxide for use in sunscreens and other products. Particles between 3-200 $\mathrm{nm}$ are used for different purposes. These particles are protective and cause minimal damage to DNA in sunlight. QDs manufactured between 3-5 nm are suitable for binding specific biomolecules.

QDs are luminescent particles, more stable than the organic dies used now and are non-toxic. 


\section{Prostheses and implants}

Nanotechnology has many applications in tissue engineering as well. Researchers put a biological material in a mould, a straitjacket as it were, which forces it to assume the shape of a body part, such as a hipbone to replace damaged or missing tissue by a similar equivalent material. Biomimetic nanostructures start with a predefined nanochemical or physical structure. A nanochemical structure may be an array of large reactive molecules attached to a surface, while a nanophysical structure may be a small crystal. Using these nanostructures as seed molecules or crystals, a material will keep growing by itself according to the researchers. Other groups want to apply nanostructured materials in artificial sensory organs such as an electronic eye, ear, or nerve.

\subsection{CONCLUSION}

Particulate colloidal carriers (i.e. liposomes or nanospheres or nanocapsules) were developed. They are now proposed as a new approach for drug administration and vaccines. Tumour blood vessels present, indeed, several abnormalities in comparison with normal physiological vessels, often including a relatively high proportion of proliferating endothelial cells, an increased tortuosity, a deficiency in pericytes and an aberrant basement membrane formation. Long circulating carriers to diffuse into the tumoural tissue because of the resulting enhanced permeability of the tumour vasculature.

During neurological diseases, BBB permeability is increased dramatically, and it has been hypothesised that drug carrier systems such as polymeric nanoparticles could cross the BBB and penetrate into the central nervous system.

PEGylated PACA nanoparticles are one such system and have been shown to dramatically penetrate the brain during experimental allergic encephalomyelitis (EAE).

In a similar manner, tamoxifen-loaded onto nanoparticles was found to be able to reduce experimental autoimmune uveitis (EAU) by entering into the ocular tissues and delivering the drug specifically to the inflammatory cells. It is also possible using those systems to improve the intracellular penetration of non-intracellularly diffusible and/or unstable compounds if the physicochemical characteristics of colloidal carriers allow certain tissues or cells to be targeted. This is illustrated by the delivery, with the aid of nanotechnologies, of antisense oligonucleotides against junction oncogenes which are found in cancers such as certain leukaemias, Ewing sarcoma and thyroid papillary carcinomas. Tumours originate from a chromosomal translocation and are therefore only found in the tumour cells, making them interesting targets. Probably because of their short biological life and limited cellular uptake, successful results have never been obtained with antisense 
oligonucleotides directed against junction genes on solid tumours in vivo. Because they are delivered to the right place in the body, nanotechnologies open new and exciting perspectives for the discovery of medicines and are more efficient. However, the preparation methods are often complex and need the use of organic solvents and surfactants that clearly represent a limitation for further medical applications.

We recently developed a new concept to obtain self-assembling nanoparticles in water without requiring any organic solvent or surfactant to overcome these inconveniences. These nanoparticles are made in a simple way, by mixing two aqueous solutions, a polymer of poly- $\beta$-cyclodextrins ( $p \beta C D$ ) and a dextran bearing alkyl side chains (DM). When these solutions are mixed, the hydrophobic alkyl chains made up of dextran spontaneously form inclusion complexes with CDs, thus forming a molecular superstructure. The structure of these nanoparticles is a core rich in CD and a corona essentially made of dextran, which sterically stabilises them. Free CD entraps drugs or other molecules inside the cores.

With nanotechnology, it is possible to achieve efficient targeting and movement of drugs across barriers. Few challenges which can be encountered will be characterisation of molecular targets and the expression of molecules specifically in the targeted tissues. Understanding the fate of the drug after being delivered to the targeted organ will further improve the efficiency of nanosystems to be used in inflammation.

In diabetes management, the use of a microphisiometer or implantable sensors allows the monitoring of insulin concentrations in real time. For the treatment of diabetes, polymeric, polysaccharide and inhalable nanoparticles can be used as carriers for the administration of insulin. Applications of nanotechnology in developments of oral insulin, the use of microspheres for oral insulin production and in development of artificial pancreas will be effective in the treatment of diabetes. With the help of nanopumps, it will be possible to administer small drug doses over a long period of time at a constant rate, which will also help in balancing the amount of sugars in blood.

Polymeric nanoparticles provide clinically useful agents for the treatment of $\mathrm{PAH}$, in which currently used potential drugs have a limitation of significantly short half-life, rendering systemic administration difficult. The use of nanoparticles in the form of liposomes, micelles, nanocrystals and nanoprecipitates can help to overcome these difficulties and may prove to be an efficient treatment for pulmonary hypertension in asthma and COPD.

All in all, the application of original physicochemical concepts to the formulation of particulate colloidal carriers may lead to efficient systems' development for the controlled administration of drugs to specific tissues, cells or even intracellular compartments. 


\section{REFERENCES}

1. C.P. Poole, F.J. Owens. Introduction to Nanotechnology, John Wiley \& Sons, Hoboken, New Jersey, USA, 2003, p. 400.

2. K.B. Rathod, M.B. Patel, P.K. Parmar, S.R. Kharadi, P.V. Patel, K.S. Patel. Int. J. Pharm. Pharm. Sci. 3 (2011) 8-12.

3. R. Kelsall, I.W. Hamley, M. Geoghegan. Nanoscale Science and Technology, Wiley, New York, USA, 2005, p. 472.

4. S.M. Moghimi, A.C. Hunter, J.C. Murray. FASEB J. 19 (2005) 11-30.

5. O.C. Farokhzad, R. Langer. ACS Nano 3 (2009) 16-20.

6. Materials used in Nanotechnology. http://en.wikipedia.org/wiki/Nanomaterials (July 20, 2007)

7. S.R. Mudshinge, A.B. Deore, S. Patil, C.M. Bhalgat. Saudi Pharm. J. 19 (2011) 129-141.

8. T. Neuberger, B. Schöpf, H. Hofmann, M. Hofmann, B. Rechenberg. J. Magn. Magn. Mater. 293 (2005) 483-496.

9. J. Chen, B. Wiley, Z.-Y. Li, D. Campbell, F. Saeki, H. Cang, L. Au, J. Lee, X. Li, Y. Xia. Adv. Mater. 17 (2005) 2255-2261.

10. S.E. Skrabalak, J. Chen, Y. Sun, X. Lu, L. Au, C.M. Cobley, Y. Xia. Acc. Chem. Res. 41(2008) 1587-1595.

11. C.M. Cobley, L. Au, J. Chen, Y. Xia. Expert Opin. Drug Deliv. 7(2010) 577-587.

12. P.H. Camargo, K.G. Satyanarayana, F. Wypych. Mater. Res. 12 (2009) 1-39.

13. I.-Y. Jeon, J.-B. Baek. Materials 3 (2010) 3654-3674.

14. R. Vasita, D.S. Katti. Int. J. Nanomed. 1(2006) 15-30.

15. B. Singhana, P. Slattery, A. Chen, M. Wallace, M.P. Melancon. AAPS Pharm. Sci. Tech. 15 (2014) 741-752.

16. J. Drbohlavova, V. Adam, R. Kizek, J. Hubalek. Int. J. Mol. Sci. 10 (2009) 656-673.

17. A.M. Smith, H. Duan, A.M. Mohs, S. Nie. Adv. Drug Deliv. Rev. 60 (2008) 1226-1240.

18. R. Bakry, R.M. Vallant, M. Najam-ul-Haq, M. Rainer, Z. Szabo, C.W. Huck, G.K. Bonn. Int. J. Nanomed. 2 (2007) 639-649.

19. P. Anilkumar, F. Lu, L. Cao, P.G. Luo, J.-H. Liu, S. Sahu, K.N. Tackett, Y. Wang, Y.-P. Sun. Curr. Med. Chem. 18 (2011) 2045-2059.

20. H. He, L.A. Pham-Huy, P. Dramou, D. Xiao, P. Zuo, C. Pham-Huy. Biomed. Res. Int. 2013 (2013) 1-12.

21. A. Hafner, J. Lovric, G.P. Lakos, I. Pepic. Int. J. Nanomed. 9 (2014) 1005-1023.

22. V. Wagner, A. Dullaart, A.K. Bock, A. Zweck. Natl. Biotech. 24 (2006) 1211-1217.

23. R. Duncan. Natl. Rev. Drug Discov. 2 (2003) 347-360.

24. W.B. Tan, Y. Zhang. J. Biomed. Mater. Res. A 75 (2005) 56-62.

25. J. Khandare, T. Minko. Prog. Polym. Sci. 31 (2006) 359-397.

26. L. Brannon-Peppas, J.O. Blanchette. Adv. Drug Deliv. Rev. 56 (2004) 1649-1659.

27. V.P. Torchilin. Adv. Drug Deliv. Rev. 54 (2002) 235-252.

28. E.S. Lee, K. Na, Y.H. Bae. Nano. Lett. 5 (2005) 325-329.

29. C.A. Lipinski. J. Pharmacol. Toxicol. Methods 44 (2000) 235-249.

30. R. Krishna, L.D. Mayer. Eur. J. Pharm. Sci. 11 (2000) 265-283.

31. I. Brigger, C. Dubernet, P. Couvreur. Adv. Drug Deliv. Rev. 54 (2002) 631-651. 
32. J. Folkman. N. Engl. J. Med. 285 (1971) 1182-1186.

33. L. Balasubramanian, A.M. Evens. Curr. Opin. Oncol. 18 (2006) 354-359.

34. S. Sengupta, D. Eavarone, I. Capila. Nature 436 (2005) 568-572.

35. E. Ruoslahti. Natl. Rev. Cancer 2 (2002) 83-90.

36. S.K. Hobbs, W.L. Monsky, F. Yuan. Proc. Natl. Acad. Sci. U.S.A. 95 (1998) 4607-4612.

37. H. Maeda. Adv. Enzyme Regul. 41 (2001) 189-207.

38. H. Hashizume, P. Baluk, S. Morikawa. Am. J. Pathol. 156 (2000) 1363-1380.

39. J. Sudimack, R.J. Lee. Adv. Drug Deliv. Rev. 41 (2000) 147-162.

40. D. Goren, A.T. Horowitz, D. Tzemach. Clin. Cancer Res. 6 (2000) 1949-1957.

41. A.R. Hilgenbrink, P.S. Low. J. Pharm. Sci. 94 (2005) 2135-2146.

42. B. Breyer, W. Jiang, H. Cheng. Curr. Gene Ther. 1 (2001) 149-162.

43. S. Lehrman. Nature 401 (1999) 517-518.

44. J.Y. Sun, V. Anand-Jawa, S. Chatterjee. Gene Ther. 10 (2003) 964-976.

45. A. El-Aneed. J. Control. Release 94 (2004) 1-14.

46. $\quad$ A.P. Rolland. Crit. Rev. Ther. Drug Carrier Syst. 15 (1998) 143-198.

47. D.J. Glover, H.J. Lipps, D.A. Jans. Natl. Rev. Genet. 6 (2005) 299-310

48. M.D. Brown, A.G. Schatzlein, I.F. Uchegbu. Int. J. Pharm. 229 (2001) 1-21.

49. R. Kircheis, T. Blessing, S. Brunner. J. Control. Release 72 (2001) 165-170.

50. M.C. Garnett. Crit. Rev. Ther. Drug Carrier Syst. 16 (1999) 147-207.

51. A.R. Klemm, D. Young, J.B. Lloyd. Biochem. Pharmacol. 56 (1998) 41-46.

52. R. Kircheis, S. Schuller, S. Brunner. J. Gene Med. 1 (1999) 111-120.

53. K. Kunath, A. von Harpe, D.J. Fischer. Control. Release 89 (2003) 113-125.

54. D. Fischer, T. Bieber, Y. Li, H.P. Elsasser, T. Kissel. Pharm. Res. 16 (1999) 1273-1279.

55. H. Petersen, P.M. Fechner, A.L. Martin. Bioconjug. Chem. 13 (2002) 845-854.

56. Y.H. Choi, F. Liu, J.S. Kim. J. Control. Release 54 (1998) 39-48.

57. Y.B. Lim, S.O. Han, H.U. Kong. Pharm. Res. 17 (2000) 811-816.

58. T.I. Kim, H.J. Seo, J.S. Choi. Bioconjug. Chem. 16 (2005) 1140-1148.

59. J. Wang, H.Q. Mao, K.W. Leong. J. Am. Chem. Soc. 123 (2001) 9480-9491.

60. M. Jones, J. Leroux. Eur. J. Pharm. Biopharm. 48 (1999) 101-111.

61. M.L. Adams, A. Lavasanifar, G.S. Kwon. J. Pharm. Sci. 92 (2003) 1343-1355.

62. R. Salvic, L. Luo, A. Eisenberg, D. Maysinger. Science 300 (2003) 615-628.

63. H. Otsuka, Y. Nagasaki, K. Kataoka. Adv. Drug Deliv. Rev. 55 (2003) 403-419.

64. Y. Akiyama, H. Otsuka, Y. Nagasaki. Bioconjug. Chem. 11 (2000) 947-950.

65. P. Wang, K.L. Tan, E.T. Kang. J. Biomater. Sci. Polym. 11 (2000) 169-186.

66. M. Yokoyama, A. Satoh, Y. Sakurai, T. Okano. J. Control. Release 55 (1998) 219-229.

67. M.L. Forrest, C.Y. Won, A.W. Malick, G.S. Kwon. J. Control. Release 110 (2006) 370-377.

68. H. Dian, 2002, M. S. Thesis. pp. 1-149.

69. C. Dufes, I.F. Uchegbu, A.G. Schatzlein. Adv. Drug Deliv. Rev. 57 (2005) 2177-2202.

70. G.J.M. Koper, M.H.P. van Genderen, E.C. Roman, M.W.P.L. Baars, E.W. Meijer, M. Borkovec . J. Am. Chem. Soc. 119 (1997) 6512-6521.

71. E.M.M. de Brabander-van den Berg, E.W. Meijer. Angew Chem. Int. Ed. Engl. 32 (1993) 1308-1311.

72. B.K. Nanjwade, H.M. Bechra, G.K. Derkara, F.V. Manvi , V.K. Nanjwad. Eur. J. Pharm. Sci. 38 (2009) 185-196. 
73. K. Lorenz, D. Holter, B. Stuhn, R. Mulhaupt, H. Frey. Adv. Mater. 8 (1996) 414-416.

74. $\quad$ H. Frey, K. Lorenz, R. Mulhaupt. Macromol. Symp. 102 (1996) 19-26.

75. J.H. Cameron, A. Facher, G. Lattermann, S. Diele. Adv. Mater. 9 (1997) 398-403.

76. U. Stebani, G. Lattermann. Adv. Mater. 7 (1995) 578-581.

77. N. Boiko, X. Zhu, A. Bobrovsky, V. Shibaev. Chem. Mater. 13 (2001) 1447-1452.

78. P. Schilrreff, C. Mundiña-Weilenmann, E.L. Romero, M.J. Morilla. Int. J. Nanomed. 7 (2012) 4121-4133.

79. T.A. Betley, J.A. Hessler, A. Mecke, M.M. Banaszak Holl, B.G. Orr, S. Uppuluri, D.A. Tomalia. Langmuir 18 (2002) 3127-3133.

80. K. Inoue, H. Sakai, S. Ochi, T. Itaya, T. Tanigaki. J. Am. Chem. Soc. 116 (1994) 10783-10784.

81. S. Ghorai, D. Bhattacharyya, A. Bhattacharjya. Tetrahedron Lett. 45 (2004) 6191-6194.

82. K. Sadler, J.P. Tam. Mol. Biotechnol. 90 (2002) 195-229.

83. G.A. Kinberger, W. Cai, M. Goodman. J. Am. Chem. Soc. 124 (2002) 15162-15163.

84. T. Darbre, J.L. Reymond. Acc. Chem. Res. 39 (2006) 925-934.

85. E.K. Woller, M.J. Cloninger. Biomacromolecules 2 (2001) 1052-1054.

86. R. Roy, M.G. Baek. J. Biotechnol. 90 (2002) 291-309.

87. R. Roy, D. Zanini, S. Meunier, A. Romanowska. Chem. Commun. (1993) 1869-1872.

88. A. Pushechnikov, A.A. Jalisatgi, M.F. Hawthorne. Chem. Commun. 49 (2013) 3579-3581.

89. H.B. Agashe, A.K. Babbar, S. Jain, R.K. Sharma, A.K. Mishra, A. Asthana, M. Garg, T. Dutta, N.K. Jain. Nanomedicine 3 (2007) 1120-1127.

90. B. Klajnert, M. Bryszewska. Cell Mol. Biol. Lett. 7 (2002) 1087-1094.

91. K.K. Ong, A.L. Jenkins, R. Cheng, D.A. Tomalia, H.D. Durst, J.L. Jensen, P.A. Emanuel, C.R. Swim, R. Yin. Anal. Chim. Acta 444 (2001) 143-148.

92. M.T. Islam, I.J. Majoros, J.R. Baker Jr. J. Chromatogr. $B$

93. C.J. Hawker, J.M.J. Frechet. J. Am. Chem. Soc. 112 (1990) 7638-7647.

94. P. Kesharwani, K. Jain, N.K. Jain. Prog. Polym. Sci. (2013) 1-125.

95. S. Svenson, D.A. Tomalia. Adv. Drug Deliv. Rev. 57 (2005) 2106-2129.

96. S. Tripathy, M.K. Das. J. App. Pharm. Sci. 3 (2013) 142-149.

97. S. Pushkar, A. Philip, K. Pathak, D. Pathak. Indian J. Pharm. Educ. Res. 40 (2006) 153-158.

98. F. Zeng, S.C. Zimmerman. Chem. Rev. 97 (1997) 1681-1712.

99. I. Brigger, C. Dubernet, P. Couvreur. Adv. Drug Deliv. Rev. 54 (2002) 631-651.

100. J.D. Kingsley. J. Neuroimmunol. Pharmacol. 1 (2006) 340-350.

101. A.V. Kabanov, E.V. Batrakova. Curr. Pharm. Des. 10 (2004) 1355-1363.

102. W. Pardridge. Arch. Neurol. 59 (2002) 35-40.

103. A. Misra. J. Pharm. Sci. 6 (2003) 252-273.

104. V. Weissig. J. Liposome Res. 16 (2006) 249-264.

105. A. Kozubek. Acta Biochim. Pol. 47 (2000) 639-649.

106. M. Voinea, M. Simionescu. J. Cell Mol. Med. 6 (2002) 465-474.

107. P. Calvo. Pharm. Res. 18 (2001) 1157-1166.

108. R. Gref. Science 263 (1994) 1600-1630.

109. B.E. Rabinow. Natl. Rev. Drug Discov. 3 (2004) 785-796.

110. V.P. Torchilin. Cell Mol. Life Sci. 61 (2004) 2549-2559. 
111. K. Kataoka, A. Harada, Y. Nagasaki. Adv. Drug. Deliv. Rev. 47 (2001) 113-131.

112. A. V. Kabanov, V. Y. Alakhov, Crit. Rev. Ther. Drug Carrier Syst., 19 (2002), $1-72$.

113. T.K. Bronich. Colloids Surf. B 16 (1999) 243-251.

114. T.K. Bronich. J. Am. Chem. Soc. 127 (2005) 8236-8247.

115. T.K. Bronich. J. Drug Target. 14 (2006) 357-366.

116. G. Che. Chem. Mater. 10 (1998) 260-270.

117. G.M. Acland, G.D. Aguirre, J. Bennett. Mol. Ther. 12 (2005) 1072-1082.

118. C.E. Thomas, A. Ehrhardt, M.A. Kay. Nat. Rev. Genet. 4 (2003) 346-358.

119. R. Farjo, J. Skaggs, A.B. Quiambao, M.J. Cooper. PLoS ONE 1 (2006) 1-38.

120. M. Mikhaylova, N. Bobrysheva, M. Osmolowsky. Langmuir 20 (2004) 2472-2477.

121. S. Rahiman, B.A. Tantry. J Nanomed Nanotechol. 3 (2012) 137-144.

122. D. Aronson. Adv Cardiol. 45 (2008) 1-16.

123. S.M. Moghimi, A.C. Hunter, J.C. Murray. FASEB J. 19 (2005) 311-330.

124. Microphysiometer using multiwalle carbon nanotubes enables constant real time monitoring of microliters of insulin.

http://nextbigfuture.com/2008/04/microphysiometer-using-multiwallcarbon.html (Oct 2, 2007).

125. A.K. Arya, L. Kumar, D. Pokharia, K. Tripathi. Dig. Nanomater. Bios. 3 (2008) 221-225.

126. P.S. Sona. Dig. Nanomater. Bios. 5 (2010) 411-418.

127. B. Sarmento, A. Ribeiro, F. Veiga, D. Ferreira, R. Neufeld. Biomacromolecules 8 (2007) 3054-3060.

128. J.S. Gordon. Diabetes Metab. Res. Rev. 18 (2002) 28-37.

129. J.K. Santosh. Acta Pharm. Sin. B 51 (2009) 121-127.

130. M.A. Libert. Diabetes Technol. Ther. 3 (2001) 431-449.

131. D. Zhang, T. Tan, L. Gao, W. Zhao, P. Wang. Drug Dev. Ind. Pharm. 33 (2007) 569-575.

132. O. Balland, H. Pinto-Alphandary, A. Viron, E. Puvion, A. Andremont, P. Couvreur. J. Antimicrob. Chemother. 37 (1996) 105-115.

133. S. Gupta, S.P. Viyas. J. Drug Target. 15 (2007) 206-217.

134. R.O. Hynes. Nat. Med. 8 (2002) 918-921.

135. X. Chen, C. Plasencia, Y. Hou, N. Neamati. J. Med. Chem. 48 (2005) 1098-1106.

136. R.M. Schiffelers, G.A. Koning, T.L. den Hagen, M.H. Fens, A.J. Schraa, A.P. Janssen, R.J. Kok, G. Molema, G. Strom. J. Control. Rel. 91 (2003) 115-122.

137. N.K. Haass, K.S. Smalley, L. Li, M. Herlyn. Pigment Cell Res. 18 (2005) 150-159.

138. W. Arap, R.R. Pasqualini, E. Ruoslahti. Science 279 (1998) 379-380.

139. S. Zhuang, R.G. Schnellmann. J. Pharmacol. Exp. Ther. 319 (2006) 991-997.

140. A.L. Dunehoo, M. Anderson, S. Majumdar, N. Kobayashi, C. Berkland, T.J. Siahaan. J. Pharma. Sci. 95 (2006) 1856-1872.

141. Applications of Nanotechnology in Chronic Diseases-I. http://nptel.ac.in/courses/118107015/module4/lecture4/lecture4.pdf (April 10, 2015).

142. N.G. Portney, M. Ozkan. Anal. Bioanal. Chem. 384 (2006) 620-630. 